TRANSACTIONS OF THE

AMERICAN MATHEMATICAL SOCIETY

Volume 353, Number 4, Pages 1279-1320

S 0002-9947(00)02704-5

Article electronically published on December 15, 2000

\title{
THE TOPOLOGICAL DYNAMICS OF SEMIGROUP ACTIONS
}

\author{
DAVID B. ELLIS, ROBERT ELLIS, AND MAHESH NERURKAR
}

\begin{abstract}
In these notes we explore the fine structure of recurrence for semigroup actions, using the algebraic structure of compactifications of the acting semigroup.
\end{abstract}

\section{INTRODUCTION}

This is a self-contained exposition of aspects of the theory of recurrence for flows $(X, T)$ where $X$ is a compact Hausdorff space and $T$ a semigroup. A good account of the case when $T$ is $\mathbb{Z}$ or $\mathbb{N}$ is to be found in $[\mathrm{F}$.

Contrary to what one might hope or expect, the passage from the group to the semigroup case is not straightforward. Many theorems do not go over, and care must be exercised in choosing the proper definitions.

Another difference between our account and Furstenberg's is the emphasis we place on the abstract or algebraic approach to the subject. The essence of this approach is that the asymptotic properties of the trajectories are captured by the algebraic properties of a suitable compactification of $T$. (Here we consider two such compactifications - the enveloping semigroup of the flow $(X, T)$ and the StoneCech compactification, $\beta T$ of $T$.)

One of the most successful applications of this approach is the deep results obtained by Furstenberg and Katznelson in their paper "Idempotents in compact semigroups and Ramsey theory" [FK]. In Section 4 we develop and generalize the algebraic machinery used in their paper (see Proposition 4.12). This proposition is the backbone of the proof of 6.3 , an abstract multi-recurrence theorem which is the basis of a unified approach to a variety of multi-recurrence results occurring in the literature (see Section 6).

A propos the algebraic approach it would be remiss on our part not to mention the profound influence the paper "Product recurrence and distal points" [AF] by Auslander and Furstenberg has had on this endeavor. In particular the ordering of the idempotents in $\mathcal{E}$-semigroups (see 4.3 ), the role played by the maximal idempotents and the notion of a cancellation semigroup originated with them. In Section 5 we generalize some of their results.

Besides studying semigroup as opposed to group flows we also wished to replace the combinatorial approach by the abstract one, to eliminate all reliance on the notion of IP sets, to replace them entirely by corresponding results about idempotents. As even a cursory glance will show, we were hardly successful in this

Received by the editors July 21, 1998 and, in revised form, January 17, 2000.

2000 Mathematics Subject Classification. Primary 54H20, 37B05.

Key words and phrases. Topological dynamics, recurrence.

(C)2000 American Mathematical Society 
endeavor. Indeed many results and constructions in Sections 3 and 5 are generalizations of the standard ones. (A prime example is Galvin's theorem 3.3.) These were used to generalize some of the work of Auslander and Furstenberg mentioned above.

We wish to thank Professor Joe Auslander, Professor Tomasz Dawnarowicz and Mr. Bartosz Frej for reading an earlier draft. Their comments, corrections and suggestions led to an improved revision.

\section{Dynamical Systems}

1.1. Definition. Let $X$ be a set and $T$ be a semigroup. A (right) $T$-action on $X$ is a mapping

$$
\begin{aligned}
\pi: X \times T & \rightarrow X \\
(x, t) & \rightarrow x t
\end{aligned}
$$

such that

$$
(x t) s=x(t s) \text {, for all } x \in X, t, s \in T \text {. }
$$

When we have a $T$-action on $X$, we shall say that $T$ acts on $X$ (via $\pi$ ). If $T$ has an identity $e$, then we also require that $x e=x$ for all $x \in X$. The symbol $\pi$ will often be suppressed.

1.2. Definition. A dynamical system or flow is a triple $(X, T, \pi)$ where $X$ is a compact, Hausdorff space, $T$ is a semigroup which acts on $X$, such that the action $\pi$ is continuous. The mapping $\pi$ will usually be supressed and the flow denoted $(X, T)$.

1.3. Remark. In the literature $T$ is usually equipped with some topology. However, the topology on $T$ is irrelevant for most of the problems with which we are concerned. Consequently we ignore it, or what amounts to the same thing, provide $T$ with the discrete topology. In this case $(X, T, \pi)$ is a flow if for each $t \in T$ the map

$$
\begin{aligned}
\pi_{t}: X & \rightarrow X \\
x & \rightarrow x t
\end{aligned}
$$

is continuous. Moreover to avoid uninteresting cases we assume that $T$ is infinite.

1.4. Definition. Given a flow (or just an action) $(X, T)$, the orbit of a point $x$ in $X$ is the set

$$
\operatorname{Orb}(x)=x T=\left\{x \pi_{t}=x t \mid t \in T\right\} .
$$

1.5. Remark. Note that when the semigroup $T$ contains an identity, $x \in x T$. In general this need not be the case. We will examine some specific examples later, but first we review some basic concepts from dynamical systems in the context of semigroups.

1.6. Definition. Let $(X, T, \pi)$ be a flow.

(1) Let $\emptyset \neq A \subseteq X$. We say that $A$ is invariant if $A T \equiv\{a t \mid a \in A, t \in T\} \subseteq A$.

(2) If $\emptyset \neq A$ is both closed and invariant, then the restriction of $\pi$ to $A \times T$ defines an action of $T$ on $A$. The resulting flow $(A, T)$ is called a subflow of the flow $(X, T)$.

(3) A point $x \in X$ is a transitive point if $\overline{x T}=X$. The flow $(X, T)$ is said to be point transitive if there exists a transitive point $x$ in $X$. 
(4) The flow $(X, T)$ is minimal if $\overline{x T}=X$ for all $x \in X$, i.e. if every point is a transitive point.

(5) Let $A$ be a non-empty, closed invariant subset of $X$. Then $A$ is a minimal set if the flow $(A, T)$ is minimal.

1.7. Proposition. Let $A$ be a non-empty, closed invariant subset of the flow $(X, T)$. Then $A$ is a minimal set if and only if $A$ is minimal with respect to the property of being non-empty, closed, and invariant.

Proof. This is immediate from the definition.

A simple application of Zorn's lemma yields

1.8. Proposition. Let $(X, T)$ be a flow. Then there exists a minimal subset $A$ of $X$.

1.9. Remark. In these notes we are considering actions by general semigroups. For certain semigroups there are no non-trivial point transitive flows. One such example is the following.

1.10. Example. Let $T$ be an infinite set. Fix an element 0 in $T$ and set $t s=0$ for all $t, s \in T$. This multiplication defines a semigroup structure on $T$ called the 0 -structure. We claim that in this case the one point flow is the only flow for which $(X, T)$ is point transitive.

Proof. Suppose that $(X, T)$ is a transitive flow with transitive point $x$. Then since $x \in \overline{x T}$, there exists a net $\left\{t_{\alpha}\right\} \in T$ with $x=\lim x t_{\alpha}$. Hence $x t=\lim x t_{\alpha} t=x 0$ for all $t \in T$. Therefore $X=\{x 0\}$ is a singleton set.

1.11. Definition. Let $(X, T)$ and $(Y, T)$ be flows and $f: X \rightarrow Y$ be a mapping. We say that $f$ is a homomorphism if

(a) $f$ is continuous, and

(b) $f(x t)=f(x) t$ for all $x \in X$ and $t \in T$.

The study of the structure and asymptotics of orbits is a central theme in the theory of dynamical systems. It is often fruitful to identify the element $t$ of $T$ with the map $\pi_{t}$ (as defined in 1.3) from $X$ to itself. The space $X^{X}$ of all self-maps of $X$ is provided with the topology of pointwise convergence. By way of illustration, note that $y \in \overline{x T}$ if and only if $y=\lim x t_{\alpha}=x \lim \pi_{t_{\alpha}}$, where the last limit is the limit of the net $\left\{\pi_{t_{\alpha}}\right\}$ in $X^{X}$. Thus one is naturally led to consider the closure of $T$ in the topology of pointwise convergence on $X^{X}$. We now proceed to examine some elementary properties of this space. We will see in the later sections that this study is an illustration of a more general principle mentioned earlier, namely "The asymptotic properties of orbits under $T$ are captured in suitable compactifications of $T$ ".

1.12. Proposition. Let $X$ be a compact Hausdorff space and $X^{X}$ the space of all self-maps of $X$ provided with the topology of pointwise convergence. Then

(1) $X^{X}$ is a compact Hausdorff semigroup under composition of maps.

(2) $X^{X}$ acts on $X$ by evaluation of maps: $(x, p) \rightarrow x p$.

(3) If a semigroup $T$ acts on $X$, then the map $t \rightarrow \pi_{t}$ is a homomorphism of semigroups $T \rightarrow X^{X}$. Moreover $(X, T)$ is a dynamical system if and only if the image of this homomorphism is contained in the set of continuous selfmappings of $X$. 
(4) If $(X, T)$ is a dynamical system, then $E(X, T) \equiv \bar{T} \equiv \overline{\left\{\pi_{t} \mid t \in T\right\}}$ is a closed subsemigroup of $X^{X}$.

Proof. The first three statements are immediate from the definitions. To prove the last statement let $q \in E(X, T)$ and $\pi_{t_{\alpha}} \rightarrow q$. Then $x t_{\alpha}=x \pi_{t_{\alpha}} \rightarrow x q$ for all $x \in X$. Thus $\left(x t_{\alpha}\right) \pi_{t} \rightarrow(x q) \pi_{t}=x\left(q \pi_{t}\right)$ for all $x \in X$. Hence $\pi_{t_{\alpha} t}=\pi_{t_{\alpha}} \pi_{t} \rightarrow q \pi_{t}$, and $q \pi_{t} \in E(X, T)$.

Now let $p \in E(X, T)$ and let $\pi_{t_{\beta}} \rightarrow p$. Then

$$
x\left(q \pi_{t_{\beta}}\right)=(x q) \pi_{t_{\beta}} \rightarrow(x q) p=x(q p), \quad \text { for all } x \in X .
$$

Thus $q \pi_{t_{\beta}} \rightarrow q p$, and $q p \in E(X, T)$. This proves that $E(X, T)$ is a subsemigroup of $X^{X}$

1.13. Remark. (1) When $T$ acts on $X$, the homomorphism $t \rightarrow \pi_{t}$ referred to in Proposition 1.12 defines a right action of $T$ on $X^{X}$ (as a subsemigroup) which extends to an action of $E(X, T)$ on $X^{X}$ when $(X, T)$ is a dynamical system.

(2) It is important to note that the map $t \rightarrow \pi_{t}$ of Proposition 1.12 need not be one-one. Indeed the image may be a singleton. Nevertheless for the study of dynamical systems nothing is lost by viewing $T$ as a subset of $E(X, T)$ (identifying $t$ with $\left.\pi_{t}\right)$.

(3) If $(X, T)$ is a dynamical system and $S$ is a subsemigroup of $T$, then $E(X, S) \subset$ $E(X, T)$.

The space $E(X, T)$ referred to in Proposition 1.12, is a compactification of the semigroup $T$. This compactification is of historical as well as practical importance. We emphasize this by

1.14. Definition. Let $(X, T, \pi)$ be a flow and $X^{X}$ the space of all self-maps of $X$ provided with the topology of pointwise convergence. We define the enveloping semigroup of the flow $(X, T)$, denoted $E(X, T)$, to be the closure of $T$ (as a subset of $\left.X^{X}\right)$.

We will also consider other compactifications of $T$, but before discussing them, we examine the enveloping semigroup a bit more closely. In fact we shall see that many of the other compactifications of $T$ have certain key properties in common with $E(X, T)$.

1.15. Proposition. Let $E(X, T)$ be the enveloping semigroup of a dynamical system $(X, T)$. Then

$(1) R_{t}: E(X, T) \rightarrow E(X, T)$ is continuous for every $t \in T$,

$$
p \quad \rightarrow \quad p t
$$

(2) $L_{p}: E(X, T) \rightarrow E(X, T)$ is continuous for every $p \in E(X, T)$.

$$
q \quad \rightarrow \quad p q
$$

(3) $T$ has a natural action on $E(X, T)$ by multiplication and this action defines a flow $(E(X, T), T)$.

(4) For each $x \in X$ the map $E(X, T) \rightarrow X$ given by $p \rightarrow x p$ is a homomorphism of dynamical systems.

Proof. (1) This follows from the fact that each $t \in T$ is continuous as a self-map of $X$, and the definition of the topology of pointwise convergence on $X^{X}$. 
(2) Let $p, q, q_{\alpha} \in E(X, T)$ and $q_{\alpha} \rightarrow q$. Then $x q_{\alpha} \rightarrow x q$ for all $x \in X$. Thus $x\left(p q_{\alpha}\right)=(x p) q_{\alpha} \rightarrow(x p) q=x(p q)$ for each $x \in X$, i.e. $p q_{\alpha} \rightarrow p q$. Thus $L_{p}$ is continuous.

(3) The $T$ action is given by $(p, t) \rightarrow p t \equiv p \pi_{t}$. The fact that $(E(X, T), T)$ is a flow follows from (1) and (3) of 1.12 .

(4) Continuity follows from the definition of the topology of pointwise convergence. The fact that $p \rightarrow x p$ is a homomorphism follows from (2) of 1.12.

1.16. Remark. (1) Since $E(X)$ is a subsemigroup of $X^{X}$, it acts on $X$ (on the right). This action extends the action of $T$ on $X$, but in general $(X, E(X))$ is not a dynamical system (the action need not be continuous) even when $(X, T)$ is a dynamical system.

(2) When $T$ has an identity $e$, it is a transitive point of the flow $(E(X), T)$.

(3) If $(X, T)$ is a dynamical system, then the map

$$
\begin{array}{ccc}
\varphi: E(X, T) & \rightarrow & E(E(X, T), T) \\
p & \rightarrow & R_{p}
\end{array}
$$

is an epimorphism of flows, where $R_{p}: E(X, T) \rightarrow E(X, T)$ takes $q$ to $q p$. When $T$ has an identity, evaluation at the identity provides an inverse of $\varphi$. More generally if the flow $(X, T)$ is point transitive, say $X=\overline{x_{0} T}$, then $R_{p_{1}}=R_{p_{2}}$ implies that $q p_{1}=q p_{2}$ for all $q \in E(X, T)$. Hence for any $x \in X, x p_{1}=x_{0} q p_{1}=x_{0} q p_{2}=x p_{2}$, so $p_{1}=p_{2}$ and $\varphi$ is one-one.

1.17. Definition. A set $E$ is an $\mathcal{E}$-semigroup if it satisfies the following three conditions:

(i) The set $E$ is a semigroup.

(ii) The set $E$ has a compact, Hausdorff topology.

(iii) The left translation map $L_{p}: E \rightarrow E, L_{p}(q)=p q$ is continuous for every $p \in E$.

This definition follows the terminology introduced by J. Auslander and H. Furstenberg in $[\mathrm{AF}$, and is motivated by Proposition 1.15 which shows that $E(X, T)$ is an $\mathcal{E}$-semigroup. Indeed we will study various compactifications of $T$, all of which turn out to be $\mathcal{E}$-semigroups. First we provide further motivation for Definition 1.17 by proving that every $\mathcal{E}$-semigroup contains idempotents.

1.18. Lemma. Let $E$ be an $\mathcal{E}$-semigroup. Then $E$ contains an idempotent, i.e. an element $u$ with $u^{2}=u$.

Proof. Consider the inductive family $\mathcal{F}=\{\emptyset \neq N \subset E \mid N \cdot N \subset N, N$ closed $\}$. Let $M \in \mathcal{F}$ be minimal with respect to inclusion, and $w \in M$. Then $M \supset w M=$ $L_{w}(M) \in \mathcal{F}$, so by the minimality of $M, w M=M$. Thus

$$
\emptyset \neq\{q \in M \mid w q=w\}=L_{w}^{-1}(w) \cap M=Q \in \mathcal{F} .
$$

By minimality $Q=M$ and since $w \in M, w^{2}=w$.

In the next section we shall study in detail perhaps the most important example of an $\mathcal{E}$-semigroup namely the Stone-Cech compactification, $\beta T$ of the semigroup $T$. In Section 3 we shall use the properties of $\beta T$ to derive versions of Galvin's and Hindman's theorems. A structure theory for the set of idempotents in an $\mathcal{E}$-semigroup will be developed in Section 4, and exploited in Section 6 to obtain 
multiple recurrence results. In Section 5 we again use the properties of $\beta T$ to derive characterizations of various types of recurrence.

\section{The Beta COMPACTIFICATION OF $T$}

Given a dynamical system with acting semigroup $T$, one of the ways to capture asymptotics and recurrence of the trajectories is to consider a suitable compactification of $T$. We saw an example of this in the previous section. The Stone-Cech compactification, $\beta T$ is a particularly good compactification to use in view of its universal properties. In this section we shall study $\beta T$ in some detail. Indeed $\beta T$ is another example of an $\mathcal{E}$-semigroup. This algebraic structure on $\beta T$ plays a crucial role throughout this exposition. We begin with a definition which characterizes $\beta T$ by its properties, and then show how its algebraic structure can be deduced from these properties. For an alternate approach see Appendix A where we give a detailed construction of $\beta T$ in terms of ultrafilters on $T$, which leads to an explicit description of the semigroup structure on $\beta T$.

2.1. Definition. Let $T$ be a set with the discrete topology. The Stone-Čech compactification $\beta T$ of $T$ is determined up to homeomorphism by the following properties:

(1) $T \subset \beta T$,

(2) $\bar{T}=\beta T$,

(3) $\beta T$ is a compact Hausdorff space,

(4) if $X$ is a compact Hausdorff space and $f: T \rightarrow X$ is any map, then $f$ has a unique extension to a continuous map $\hat{f}: \beta T \rightarrow X$.

2.2. Remark. (1) $\{\bar{A} \mid A \subset T\}$ is a base for the topology on $\beta T$.

(2) $\beta T=\bar{A} \cup \overline{T \backslash A}$ is a disjoint union for every $A \subset T$. Thus $\bar{A}$ is both open and closed.

(3) $p \in \beta T \backslash T$ if and only if every neighborhood of $p$ contains infinitely many elements of $T$.

(4) The closure of every open subset of $\beta T$ is open.

(5) If $\emptyset \neq H \subset T$, then the extension of the inclusion to a map $\beta H \rightarrow \beta T$ is a homeomorphism of $\beta H$ with $\bar{H} \subset \beta T$. To see this consider the map

$$
T=H \uplus T \backslash H \rightarrow \beta H \uplus\{\text { point }\} ;
$$

it has a continuous extension to a map $\beta T \rightarrow \beta H \cup\{$ point $\}$. The restriction of this extension to $\bar{H}$ gives the inverse of the mapping above. We will often identify $\beta H$ with $\bar{H}$ in this case.

2.3. Proposition. Let $T$ be a semigroup. Then the semigroup structure on $T$ extends to a semigroup structure on $\beta T$,

$$
\begin{array}{cl}
\beta T \times \beta T & \rightarrow \beta T \\
(p, q) & \rightarrow p q
\end{array}
$$

which satisfies

(1) $R_{t}: \beta T \rightarrow \beta T$ is continuous for every $t \in T$,

$$
p \rightarrow p t
$$


(2) $L_{p}: \beta T \rightarrow \beta T$ is continuous for every $p \in \beta T$.

$$
q \quad \rightarrow \quad p q
$$

In particular $\beta T$ is an $\mathcal{E}$-semigroup.

Proof. The map $R_{t}$ is the unique continuous extension of right multiplication by $t$, and $L_{p}$ is the unique continuous extension of the map $t \rightarrow p t$ from $T$ to $\beta T$. The semigroup structure is then given by $p q \equiv L_{p}(q)$ for all $p, q \in \beta T$.

To see that $(p q) r=p(q r)$ for all $p, q, r \in \beta T$, first note that for any $s, t \in T$, the maps

$$
\begin{aligned}
\beta T & \rightarrow \beta T \\
p & \rightarrow(p s) t
\end{aligned} \text { and } \begin{array}{rll}
\beta T & \rightarrow & \beta T \\
p & \rightarrow & p(s t)
\end{array}
$$

are both continuous extensions of the map

$$
\begin{array}{ccc}
T & \rightarrow & \beta T \\
t^{\prime} & \rightarrow & \left(t^{\prime} s\right) t=t^{\prime}(s t) .
\end{array}
$$

Thus by the uniqueness of such extensions, $p(s t)=(p s) t$. Similarly the maps

$$
\begin{aligned}
\beta T & \rightarrow \beta T \\
q & \rightarrow(p q) t
\end{aligned} \text { and } \begin{array}{rll}
\beta T & \rightarrow & \beta T \\
q & \rightarrow & p(q t)
\end{array}
$$

are both continuous extensions of the map

$$
\begin{array}{ccc}
T & \rightarrow & \beta T \\
s & \rightarrow & (p s) t=p(s t) .
\end{array}
$$

Therefore $(p q) t=p(q t)$ for all $p, q \in \beta T$ and $t \in T$. Once again the maps

$$
\begin{array}{cccccc}
\beta T & \rightarrow & \beta T \\
r & \rightarrow(p q) r
\end{array} \text { and } \begin{array}{cll}
\beta T & \rightarrow & \beta T \\
r & \rightarrow & p(q r)
\end{array}
$$

are both continuous extensions of the map

$$
\begin{array}{ccc}
T & \rightarrow & \beta T \\
t & \rightarrow & (p q) t=p(q t) .
\end{array}
$$

2.4. Corollary. Let $X \times T \rightarrow X$ be a dynamical system and $x \in X$. Then the map

$$
\begin{array}{ll}
T & \rightarrow X \\
t & \rightarrow
\end{array}
$$

has a unique continuous extension to a homomorphism of dynamical systems

$$
\begin{array}{ccc}
\beta T & \rightarrow & X \\
p & \rightarrow & x p .
\end{array}
$$

Proof. That the map $p \rightarrow x p$ exists and is continuous follows immediately from the definition of $\beta T$. The fact that $x(p q)=(x p) q$ for all $p, q \in \beta T$ (and in particular that $p \rightarrow x p$ is a homomorphism of dynamical systems) follows from the fact that the maps $p \rightarrow x(p t)$ and $p \rightarrow(x p) t$ are both continuous extensions of $s \rightarrow x(s t)=$ $(x s) t$. 
2.5. Remark. (1) If the assumption that $(X, T)$ is a dynamical system is weakened to read that $T$ acts on $X$, then the unique continuous map $p \rightarrow x p$ referred to in Corollary 2.4 will not necessarily respect the action of $T$.

(2) If $(X, T)$ is a dynamical system, then so is $\left(X^{X}, T\right)$. Applying Corollary 2.4 to the latter we obtain a homomorphism $\varphi: \beta T \rightarrow X^{X}$. The homomorphism $\varphi$ is an extension of the map $t \rightarrow \pi_{t}$, so that $\varphi(p)=\lim \pi_{t_{\alpha}}$ for any $p=\lim t_{\alpha} \in$ $\beta T$. In this case $\varphi$ is not only a homomorphism of dynamical systems, but it is also a homomorphism of semigroups. It is immediate that the image of this homomorphism is the enveloping semigroup $E(X, T)$.

(3) If $T$ has an identity $e$, then the dynamical system $(\beta T, T)$ is point transitive (the orbit of $e$ is dense in $\beta T$ ). In this case it follows from Corollary 2.4 that any flow $(X, T)$ which is pointed by $x \in X$, (i.e. $\overline{x T}=X)$ is a homomorphic image of $(\beta T, T)$. In this sense $(\beta T, T)$ is universal for pointed flows with acting semigroup $T$. In particular it follows immediately that $E(\beta T) \cong \beta T$ both as dynamical systems and as semigroups. It is important to note that this need not be the case when $T$ does not contain an identity. One illustration of this is provided by Example 2.6 below.

2.6. Example. Let $T$ be an infinite set. Define $t s=t$ for all $t, s \in T$. This multiplication defines a semigroup structure on $T$ which we call the right-identitystructure.

It follows as in the proof of 2.4 that with this semigroup structure, $p q=p$ for all $p, q \in \beta T$. Note also that for every $t \in T$, the map $\pi_{t}: p \rightarrow p t=p$ is the identity map on $\beta T$. Therefore when $T$ is given the right-identity-structure, the orbit of each $p \in \beta T$ is $\{p\}$, and $\{i d\}=E(\beta T) \neq \beta T$.

2.7. Example. Let $T$ be an infinite set. Define $t s=s$ for all $t, s \in T$. This multiplication defines a semigroup structure on $T$ which we call the left-identitystructure.

With this structure $p q=q$ for every $p, q \in \beta T$, every $p \in \beta T$ has a dense orbit, and $E(\beta T) \cong \beta T$.

2.8. Remark. In Example 2.6 every singleton point is a minimal subset of $\beta T$, so there are minimal sets in $\beta T \backslash T$ and in $T$. In Example 2.7 the only minimal set is $\beta T$ itself, so there is a minimal set which intersects both $T$ and $\beta T \backslash T$. When $T$ is commutative neither of these phenomena can occur. We will show in Proposition 6.9 that if $T$ is commutative, either all the minimal sets in $\beta T$ lie in $\beta T \backslash T$, or there is only one minimal set in $\beta T$, and it is finite and lies in $T$.

2.9. Remarks. (i) The following conditions serve as assumptions in many of the results which follow.

(1) $\beta T \backslash T$ is a semigroup.

(2) $\beta T \backslash T$ contains an invariant set.

(3) $\beta T \backslash T$ is invariant.

Note that (3) implies (2) and (3) implies (1). It follows immediately from Proposition 2.3 and Lemma 1.18 that $\beta T$ always contains idempotents. Similarly assumption (1) guarantees that $\beta T \backslash T$ contains an idempotent. Assumption (3) is easily verified when $T$ is a group. In the next proposition we give conditions on $T$ which are equivalent to (3).

(ii) It is important to note that condition (1) above does not hold in general. We present an illustration of this in the following example. 
2.10. Example. Let $T$ be an infinite set with the 0-structure (as defined in 1.10).

We claim that with this semigroup structure, $p q=0$ for all $p, q \in \beta T$. In particular 0 is the only idempotent in $\beta T$, so $\beta T \backslash T$ contains no idempotents, and is not $T$-invariant.

To prove our claim we first note that for every $s \in T, R_{s}$ is the unique continuous extension of the constant map $T \rightarrow\{0\}$, thus $p s=R_{s}(p)=0$ for all $p \in \beta T$. It follows that for every $p \in \beta T, L_{p}$ is the constant map $\beta T \rightarrow\{0\}$. This shows that $p q=0$ for all $p, q \in \beta T$.

2.11. Proposition. Let $T$ be an infinite semigroup. Then $\beta T \backslash T$ is invariant if and only if $R_{t}^{-1}\{s\}$ is finite for all $t, s \in T$.

Proof. Assume that $R_{t}^{-1}\{s\}$ is finite for every $s, t \in T$ and suppose that $\beta T \backslash T$ is not invariant. Let $p \in \beta T \backslash T$ and $t, s \in T$ be such that $p t=s$. Then $p \in R_{t}^{-1}\{s\}$, so $R_{t}^{-1}\{s\}$ is a neighborhood of $p$. This contradicts the fact that every neighborhood of $p$ contains infinitely many elements of $T$.

Conversely, assume that $R_{t}^{-1}\{s\}$ is infinite. Let $p \in \beta T$ be a limit point of $R_{t}^{-1}\{s\}$. Then $p \notin T$, and by the continuity of $R_{t}, p t=s$. This shows that $\beta T \backslash T$ is not $T$-invariant.

\section{Galvin's and Hindman's Theorems for Semigroups}

In this section we derive the classical results of Galvin and Hindman by exploiting the algebraic structure of $\beta T$. When $T$ is a group, Galvin's theorem can be interpreted as saying that an open set $V \subset \beta T$ contains an idempotent in $\beta T \backslash T$ if and only if $V$ contains an infinite so-called IP-set (see the definition below). For a general semigroup any neighborhood of an idempotent in $\beta T \backslash T$ (if such an idempotent exists) contains an infinite IP-set. On the other hand, given a subset $V \subset T$ containing an IP-set, there is no guarantee that $\bar{V}$ contains an idempotent in $\beta T \backslash T$. This, however, is true under the additional assumption that $\beta T \backslash T$ is a semigroup. We analyze and give elementary proofs of these statements using the properties of $\beta T$ which were deduced in the previous section. We then deduce Hindman's theorem on finite colorings.

3.1. Definition (IP-Sets). Let $T$ be an infinite semigroup, and $S: \mathbb{N} \rightarrow T$, be a map from the set of positive integers into $T$. We shall write $S(n) \equiv S_{n}$ for the image of $n$ under $S$, and

$$
S_{F}=S_{i_{k}} \cdot S_{i_{k-1}} \cdots S_{i_{1}}
$$

for any finite ordered set $F=\left\{i_{1}<i_{2}<\cdots<i_{k}\right\} \subset \mathbb{N}$. We denote by

$$
\pi(S)=\left\{S_{F} \mid F \in \mathcal{F}\right\},
$$

where $\mathcal{F}$ is the set of all finite ordered subsets of $\mathbb{N}$. The set $\pi(S)$ is called the $I P$-set generated by $S$. Sets of the form $\pi(S)$ will be referred to as IP-sets. We set

$$
\begin{aligned}
S^{n}: & \mathbb{N} \\
i & \rightarrow S^{n}(i) \equiv S(n+i)=S_{n+i}
\end{aligned}
$$

for all $n \in \mathbb{N}$, so that

$$
\pi\left(S^{n}\right)=\left\{S_{F} \mid F \text { is an ordered subset of }\{n+1, n+2, \ldots\}\right\} .
$$


3.2. Remark. (1) From our point of view the most interesting IP-sets are the infinite ones. Even when $T$ is a group, however, it may contain many finite IP-sets. For example, $\pi(S)$ is finite when the image of $S$ is contained in a finite subgroup of $T$. The simplest example occurs when $S(n)=e$ for all $n \in \mathbb{N}$; clearly $\pi(S)=\{e\}$ in this case. Similarly when $T$ is an arbitrary semigroup the singleton $\{u\}$ is an IP-set for any idempotent $u \in T$.

(2) We will often wish to either assume or conclude that an IP-set is infinite. It is useful to observe that $\pi(S)$ is infinite if and only if $\pi\left(S^{n}\right)$ is infinite for all $n$. This follows immediately from the fact that

$$
\pi(S)=\bigcup_{F \subset\{1,2, \ldots, n\}} S_{F}\left(\pi\left(S^{n}\right)\right) .
$$

(3) It is clear that if the map $S$ is one to one, then the IP-set $\pi(S)$ is infinite. On the other hand, if $\pi(S)$ is infinite, then there exists a one to one map $\hat{S}: \mathbb{N} \rightarrow T$ such that $\pi(\hat{S}) \subset \pi(S)$. To construct $\hat{S}$, set $\hat{S}_{1}=S_{1}$ and assume that distinct elements $\hat{S}_{1}, \ldots, \hat{S}_{k}$ of $T$ have been chosen so that $\hat{S}_{F} \in \pi(S)$ for all $F \subset\{1,2, \ldots, k\}$. Now there exists $n \in \mathbb{N}$ such that

$$
\left\{\hat{S}_{G} \mid G \subset\{1,2, \ldots, k\}\right\} \subset\left\{S_{F} \mid F \subset\{1,2, \ldots, n-1\}\right\}
$$

and we can choose $\hat{S}_{k+1}=S_{Q} \in \pi\left(S^{n}\right)$ with $\hat{S}_{k+1} \notin\left\{\hat{S}_{1}, \ldots, \hat{S}_{k}\right\}\left(\pi\left(S^{n}\right)\right.$ is infinite by the previous remark). Then for any $G \subset\{1,2, \ldots, k\}, \hat{S}_{k+1} \hat{S}_{G}=S_{Q} S_{F} \in \pi(S)$ for some $F \subset\{1,2, \ldots, n-1\}$. This completes the induction step.

The following theorem of Galvin has been proved many times over (see Lemma 2.1 of $[\mathrm{BH}]$, for example). However it is worth writing the proof in this generality.

3.3. Theorem (Galvin). Let $T$ be an infinite semigroup.

(I) Let $u^{2}=u \in \beta T \backslash T$ be an idempotent, and let $\left\{V_{i} \mid i \in \mathbb{N}\right\}$ be a collection of neighborhoods of $u$. Then there exists a one to one map $S: \mathbb{N} \rightarrow T$ such that $\pi\left(S^{n}\right) \subset V_{n}$ for all $n \in \mathbb{N}$. In particular any neighborhood of $u$ contains an infinite IP-set.

(IIa) The closure of any IP-set $A \subset T$ contains an idempotent.

(IIb) Let $H$ be a subsemigroup of $T$ such that $\beta H \backslash H \equiv \bar{H} \backslash H \subseteq \beta T$ is a nonempty semigroup. Then for any infinite IP-set $A \subseteq H$, there exists an idempotent $u \in \bar{A} \cap \beta H \backslash H \subseteq \beta T \backslash T$.

3.4. Remark. (1) The proof of part (I) of this theorem involves constructing an IPset. This construction procedure is common to the earlier proofs of several multirecurrence theorems, cf. $[\mathrm{F}]$. In fact this view of Galvin's result is an abstract unification of many such arguments.

(2) Notice that (IIa) does not guarantee that the idempotent lies in $\beta T \backslash T$; indeed as we mentioned in 3.2,A $=\{u\}$ is an IP-set when $u \in T$ is an idempotent. For many of the applications we need the idempotent to be in $\beta T \backslash T$. Hence (IIb) and its requirement that the IP-set $\pi(S)$ be infinite. However this condition alone does not guarantee the existence of an idempotent in $\beta T \backslash T$. The 0 -structure on any infinite set $T$ provides a counterexample. We need to impose some additional conditions on $T$. Notice that if $\beta T \backslash T$ is a semigroup, then it contains an idempotent. In this case we obtain the desired conclusion from (IIb) by taking $H=T$.

The following lemma is used in the proof of 3.3 (I) and will also be referred to later. 
3.5. Lemma. Let $u \in \beta T \backslash T$ be an idempotent. Assume that $t_{1}, \ldots, t_{k} \in T$, and $V_{0}, V_{1}, \ldots, V_{k}$ are subsets of $T$ which satisfiy the following conditions:

(i) $u \in \overline{V_{0}}$ and

(ii) $t_{i_{m}} \cdots t_{i_{1}} t_{i} \in L_{u}^{-1}\left(\overline{V_{i}}\right)$ for all $1 \leq i<i_{1}<\cdots<i_{m} \leq k$.

Then there exists $t \in V_{0} \cap L_{u}^{-1}\left(\overline{V_{0}}\right)$ such that $t t_{i_{m}} \cdots t_{i_{1}} t_{i} \in V_{i} \cap L_{u}^{-1}\left(\overline{V_{i}}\right)$ for all $1 \leq i<i_{1}<\cdots<i_{m} \leq k$.

Proof. Condition (ii) implies that $u t_{i_{m}} \cdots t_{i_{1}} t_{i}=u t_{F} \in \overline{V_{i}}$ for all $F=\left\{i<i_{1}<\right.$ $\left.\cdots<i_{m}\right\} \subset\{1,2, \ldots, k\}$. Equivalently $u \in R_{t_{F}}^{-1}\left(\overline{V_{i}}\right)$. Combining this with assumption (i) we obtain

$$
u \in \overline{V_{0}} \cap\left(\bigcap_{i=1}^{k}\left(\bigcap_{F=\left\{i<i_{1}<\cdots<i_{m}\right\}} R_{t_{F}}^{-1}\left(\overline{V_{i}}\right)\right) \equiv W .\right.
$$

Using the assumption that $u^{2}=u$ we get that $L_{u}(u)=u^{2} \in W$, in other words $u \in L_{u}^{-1}(W) \cap W$. Now each of the sets $\overline{V_{0}}, \overline{V_{1}}, \ldots, \overline{V_{k}}$ are both open and closed in $\beta T$. Thus it follows from the continuity of $R_{t_{F}}$ and $L_{u}$, that the set $L_{u}^{-1}(W) \cap W$ is also open and closed in $\beta T$. But $u \notin T$, so there exists $u \neq t \in T \cap L_{u}^{-1}(W) \cap W$. We then have $t \in W$ and $u t \in W$, which implies

$$
t \in V_{0}, t t_{F}=t t_{i_{m}} \cdots t_{i_{1}} t_{i} \in V_{i}, \quad \text { and } \quad u t \in \overline{V_{0}}, u t t_{F} \in \overline{V_{i}}
$$

for all $F=\left\{i<i_{1}<\cdots<i_{m}\right\} \subset\{1,2, \ldots, k\}$ as desired.

Proof of Theorem 3.3. (I) We may assume without loss of generality that each $V_{i}$ is the closure of a subset of $T$ and that the $V_{i}$ are a decreasing sequence of neighborhoods of $u$. Since $u^{2}=u, L_{u}(u)=u^{2} \in V_{1}$ so $u \in V_{1} \cap L_{u}^{-1}\left(V_{1}\right)$. Since the latter is both open and closed in $\beta T$, and $u \notin T$, we can choose $S_{1} \in T$ with $S_{1} \in V_{1} \cap L_{u}^{-1}\left(V_{1}\right)$. Assume that a one-one map $S:\{1,2, \ldots, k\} \rightarrow T$ has been defined so that

$$
S_{F} \in V_{i} \cap L_{u}^{-1}\left(V_{i}\right) \text { for all } F=\left\{i<i_{1}<i_{2}<\cdots<i_{m}\right\} \subset\{1,2, \ldots, k\} .
$$

Now $u \notin T$, so $L_{u}(u)=u \in V_{k+1} \backslash\left\{S_{1}, S_{2}, \cdots, S_{k}\right\} \equiv \bar{V}_{0}$. Hence $u \in \bar{V}_{0} \cap L_{u}^{-1}\left(\bar{V}_{0}\right)$. Using 3.5 we choose $S_{k+1}$ so that

$$
S_{k+1} \in V_{0} \cap L_{u}^{-1}\left(\bar{V}_{0}\right)
$$

and $S_{k+1} S_{F} \in V_{i} \cap L_{u}^{-1}\left(V_{i}\right)$ for all $F=\left\{i<i_{1}<i_{2}<\cdots<i_{m}\right\} \subset\{1,2, \ldots, k\}$. Then $S_{1}, S_{2}, \cdots, S_{k}, S_{k+1}$ are distinct and $S_{F} \in V_{i} \cap L^{-1}\left(V_{i}\right)$ for all $F=\left\{i<i_{1}<\right.$ $\left.i_{2}<\cdots<i_{m}\right\} \subset\{1,2, \ldots, k+1\}$. Induction on $k$ now guarantees the existence of a one-one map $S: \mathbb{N} \rightarrow T$ such that $\pi\left(S^{n}\right) \subset V_{n}$ for all $n \in \mathbb{N}$.

(IIa) Let $\pi(S) \subseteq A$ be an IP-set. Let

$$
\pi\left(S^{n}\right)=\left\{S_{F} \mid F \text { an ordered subset of }\{n+1, n+2, \ldots\}\right\},
$$

as in Definition 3.1. Set

$$
\pi_{\infty}(S)=\bigcap\left\{\overline{\left(\pi\left(S^{n}\right)\right)} \mid n \in \mathbb{N}\right\}
$$

We claim that $\pi_{\infty}(S)$ is a closed non-empty subsemigroup of $\beta T$. (We shall call this semigroup the asymptotic semigroup of the IP set $\pi(S)$.) Notice that since $\pi\left(S^{n}\right) \subseteq \pi\left(S^{m}\right)$ for $n \geq m$, the compactness of $\beta T$ implies that $\pi_{\infty}(S)$ is closed and non-empty.

Let $t \in \pi\left(S^{m}\right)$. Then there exists $n \geq m$ such that $\pi\left(S^{n}\right) t \subseteq \pi\left(S^{m}\right)$. Hence $\pi_{\infty}(S) t \subseteq \overline{\pi\left(S^{n}\right)} t \subseteq \overline{\pi\left(S^{m}\right)}$. Now let $p, q \in \pi_{\infty}(S)$. Fix $m \in \mathbb{N}$, and let $t_{\alpha} \in \pi\left(S^{m}\right)$ 
with $t_{\alpha} \rightarrow q$. Then $p t_{\alpha} \in \pi_{\infty}(S) t_{\alpha} \subseteq \overline{\pi\left(S^{m}\right)}$, and hence $p q \in \overline{\pi\left(S^{m}\right)}$. Since $m$ was arbitrary, $p q \in \pi_{\infty}(S)$. This proves that $\pi_{\infty}(S)$ is a semigroup. Thus by 1.18 there exists an idempotent $u \in \pi_{\infty}(S) \subseteq \bar{A}$.

(IIb) Let $\pi(S)=A \subset H$ and construct $\pi_{\infty}(S)$ as in the proof of (IIa). Since $\pi(S)$ and hence each $\pi\left(S^{n}\right)$ is infinite (see 3.2), and $\overline{\pi\left(S^{n}\right)} \subset \bar{A} \subset \beta H$, we see that $\left\{\overline{\pi\left(S^{n}\right)} \cap(\beta H \backslash H) \mid n \in \mathbb{N}\right\}$ is a nested sequence of non-empty subsets of $\beta H \backslash H$. The compactness of $\beta H \backslash H$ now guarantees that $K \equiv \pi_{\infty}(S) \cap(\beta H \backslash H) \neq \emptyset$. The set $K$ is a closed semigroup since it is the intersection of two closed semigroups. Therefore $K$ contains an idempotent by 1.18 .

Now we derive some consequences of Galvin's theorem and its proof.

3.6. Corollary. Let $T$ be an infinite semigroup such that $\beta T \backslash T$ is a subsemigroup of $\beta T$. Then $p \in \beta T \backslash T$ is in the closure of the set of idempotents in $\beta T \backslash T$ if and only if every neighborhood of $p$ contains an infinite IP-set.

Proof. This follows immediately from 3.3.

3.7. Corollary. Let $T$ be a semigroup (group). Let

$C=\left\{p \in \beta T \mid p^{2}=p \in \beta H\right.$ for some countable subsemigroup (group) $H$ of $\left.T\right\}$.

Then $C$ is dense in the set $J(\beta T)$ of all idempotents in $\beta T$. Furthermore if $\beta T \backslash T$ is a semigroup, in particular if $\beta T \backslash T$ is invariant, then in the previous statement $\beta T$ can be replaced by $\beta T \backslash T$.

Proof. Let $p \in J(\beta T)$ and $\bar{A}$ be a neighborhood of $p$ where $A \subset T$. If $p \in T$, then $\{p\}$ is a subsemigroup of $T$ whence $p \in C$. If $p \in \beta T \backslash T$, then by $3.3, A$ contains an IP-set $\pi(S)$. Let $H$ be the subsemigroup (group) generated by the image of $S$. Then $H$ is countable and $\pi_{\infty}(S) \subseteq \beta H \cap \bar{A}$. Since $\pi_{\infty}(S)$ contains an idempotent in $\beta T$, the set $\beta H \cap \bar{A} \cap J(\beta T)$ is non-empty. Thus $p$ is in the closure of $C$.

If $\beta T \backslash T$ is a semigroup, the corresponding statement is proved by considering $\pi_{\infty}(S) \cap(\beta T \backslash T)$ instead of $\pi_{\infty}(S)$.

3.8. Proposition (Hindman). Let $T$ be an infinite semigroup. Assume also that $J(\beta T) \cap(\beta T \backslash T)$ is non-empty. Let $f$ be a map of $T$ into a finite set $F$. Then there exists an infinite IP-set $A \subseteq T$ such that $f$ is constant on $A$.

Thus in any finite coloring of $\mathbb{N}$ or $\mathbb{Z}$ there exists a monochromatic IP-set. This is usually called Hindman's theorem.

Proof. Since the finite set $F$ is a compact Hausdorff space, there is a unique continuous extension $\hat{f}$, of $f$ to $\beta T$. Let $u^{2}=u \in \beta T \backslash T$. Since $\hat{f}$ is continuous we can find a neighborhood $V$ of $u$ in $\beta T$ such that $\hat{f}$ is constant on $V$. Now apply Galvin's theorem to obtain an infinite IP-set contained in $V$.

3.9. Notation. Now we shall generalize this result of N. Hindman. To this end let $T$ be an infinite set provided with two binary operations + and $\star$ such that

(1) $(T,+)$ and $(T, \star)$ are semigroups and

(2) $\left(t_{1}+t_{2}\right) \star s=t_{1} \star s+t_{2} \star s$, for all $t_{1}, t_{2}, s \in T$.

As before these operations can be extended to $\beta T$ to provide two semigroup structures $(\beta T,+)$ and $(\beta T, \star)$ on $\beta T$. Note that even though the first structure is written additively, we do not assume that $t+s=s+t$. Let $J(\beta T,+)$ and $J(\beta T, \star)$ denote the idempotents in $(\beta T,+)$ and $(\beta T, \star)$ respectively. 
3.10. Lemma. With the notation as in 3.9, we have

(i) $\left(p_{1}+p_{2}\right) \star t=p_{1} \star t+p_{2} \star t, \quad p_{1}, p_{2} \in \beta T, t \in T$,

(ii) $J(\beta T,+) \star T \subseteq J(\beta T,+)$,

(iii) $\overline{J(\beta T,+)} \star T \subseteq \overline{J(\beta T,+)}$,

(iv) If $(\beta T \backslash T) \star T \subseteq(\beta T \backslash T)$, then $[\overline{J(\beta T,+)} \cap(\beta T \backslash T)] \star T \subseteq \overline{J(\beta T,+)} \cap(\beta T \backslash T)$.

Proof. The map $s \rightarrow s \star t:(T,+) \rightarrow(T,+)$ is a homomorphism, whence it extends to a homomorphism of $(\beta T,+)$ into $(\beta T,+)$. This implies (i). Then (ii), (iii), (iv) follow immediately from (i) and the continuity properties of $(\beta T, \star)$.

\subsection{Proposition (Hindman). Let}

(1) $(T,+, \star)$ be as in 3.9,

(2) $J(\beta T,+) \cap(\beta T \backslash T)$ be non-empty and $(\beta T \backslash T) \star T \subseteq(\beta T \backslash T)$,

(3) $f$ be a map of $T$ into a finite set $F$.

Then there exist $A_{+}, A_{\star} \subseteq T$ such that $A_{k}$ contains an IP-set with respect to the operation $k$ and $f$ is constant on $A_{+} \cup A_{\star}, k \in\{+, \star\}$.

It is easy to see that a ring without 0-divisors satisfies the conditions of 3.11 . Thus a finite coloring of the integers will possess a monocromatic set which will contain subsets $A_{+}, A_{\star}$ that are IP with respect to addition and multiplication respectively.

Proof. Since $\overline{J(\beta T,+)} \cap(\beta T \backslash T)$ is a non-empty subsemigroup of $(\beta T, \star)$, there exists a 'multiplicative' idempotent $\mu_{\star} \star \mu_{\star}=\mu_{\star} \in \overline{J(\beta T,+)} \cap(\beta T \backslash T)$. Now $F$ is finite, so there exists a neighborhood $\bar{V}$ of $\mu_{\star}$ on which the extension $\hat{f}$ of $f$ to $\beta T$ is constant. Since $\mu_{\star} \in \overline{J(\beta T,+)} \cap(\beta T \backslash T)$, there exists an 'additive' idempotent $\mu_{+}=\mu_{+}+\mu_{+} \in \bar{V}$. Now the conclusion follows from Galvin's theorem.

\section{The Structure of $\mathcal{E}$-Semigroups}

In sections 1 and 2 we studied some examples of compactifications of the acting semigroup $T$. As mentioned before, these compactifications are $\mathcal{E}$-semigroups, (recall Definition 1.17). In this section we shall develop a structure theory of such semigroups. When $E$ is an $\mathcal{E}$-semigroup we denote by $J(E)$, the set of idempotents in $E$. Note that $J(E)$ is non-empty by Lemma 1.18 .

4.1. Definition. Let $E$ be an $\mathcal{E}$-semigroup. A non-empty subset $I \subseteq E$ is a right ideal if it is closed and $I E \subseteq I$. A minimal right ideal is a right ideal that does not contain any proper right ideal of $E$. Similarly one defines a left ideal and a two-sided ideal.

The following proposition describes the structure of minimal right ideals in an $\mathcal{E}$-semigroup.

\subsection{Proposition. Let}

(1) E be an $\mathcal{E}$-semigroup,

(2) I a minimal right ideal,

(3) $J(I)=\left\{v \in I \mid v=v^{2}\right\}$ the set of idempotents in $I$.

Then,

(i) $J(I)$ is non-empty,

(ii) $v p=p$ for all $v \in J(I)$ and $p \in I$, 
(iii) $I=\bigcup\{I v \mid v \in J(I)\}$ where the union is a disjoint one and each set Iv is a group with identity $v$,

(iv) $q I$ is a minimal right ideal for all $q \in E$.

Proof. (i) Since $I$ itself is an $\mathcal{E}$-semigroup, this follows from Lemma 1.18.

(ii) Let $v \in J(I)$ and $p \in I$. Then $v I$ is a right ideal and $v I \subseteq I$. Hence $v I=I$, ( $I$ is minimal). Thus $v q=p$ for some $q \in I$. Therefore, $v p=v(v q)=v^{2} q=v q=p$.

(iii) We first show that $I v$ is a group with identity $v$. Since $v=v v \in I v$, (ii) implies that $v$ is a left identity. Let $q=p v \in I v$ with $p \in I$. Then

$$
q v=(p v) v=p v^{2}=p v=q .
$$

Thus $v$ is a two-sided identity in $I v$.

Let $q \in I v$. Since $q \in I$ and $I$ is minimal, $q I=I$. Thus, $q r=v$ for some $r \in I$. Therefore $q r v=v v=v$. Hence $r v \in I v$ is a right inverse of $q$ with respect to $v$. Applying this argument to $r v$, we obtain $x \in I v$ such that $(r v) x=v$. Then using (ii) we get

$$
q=q v=q(r v x)=(q r v) x=v x=x .
$$

Therefore $(r v) q=(r v) x=v$, i.e. $r v$ is both a left and a right inverse of $q$. Thus $I v$ is a group with identity $v$.

We now show that $\{I v \mid v \in J(I)\}$ forms a partition of $I$. Let $p \in I$, then since $p I=I$ the set $K=\{q \in I \mid p q=p\}$ is a non-empty $\mathcal{E}$-semigroup. Thus there exists an idempotent $v=v^{2} \in J(K) \subseteq J(I)$ such that $p v=p$. This shows that $I=\bigcup\{I v \mid v \in J(I)\}$.

Now let $p \in I v \cap I u,(v, u \in J(I))$. Then since $I v$ is a group there exists a $q \in I v$ such that $q p=v$. This implies that $v \in I p \subset I(I u)=I u$. But $I u$ is a group, so the idempotent $v$ must equal the identity $u \in I u$.

(iv) Let $q \in E$. It is clear that $q I=L_{q}(I)$ is a right ideal. Furthermore $L_{q}^{-1}(K)$ is a right ideal for any right ideal $K$. The fact that $q I$ is minimal now follows from the assumption that $I$ is minimal.

4.3. Definition. Following H. Furstenberg and J. Auslander, AF we introduce a quasi-order (a reflexive, transitive relation) $<$ on the set $J(E)$ of idempotents of $E$ by defining $v<u$ if and only if $v u=v$.

If $v<u$ and $u<v$ we say that $u$ and $v$ are equivalent and write $u \sim v$. It is easy to verify that $\sim$ is an equivalence relation on $J(E)$.

An idempotent $u \in J(E)$ is maximal if $v \in J(E)$ and $u<v$ implies $v<u$. One defines minimal idempotents similarly.

4.4. Lemma. Let $u \in J(E)$ and $I$ be a right ideal. Then $u I$ contains an idempotent $\theta$ such that $\theta<u$.

Proof. Note that $u I$ is a right ideal and hence has an idempotent $\omega$. Let $\omega=u v$ with $v \in I$. Set $\theta=\omega u=u v u \in u I$. Then,

$$
\theta^{2}=(u v u)(u v u)=u v\left(u^{2}\right) v u=(u v)(u v) u=\omega^{2} u=\omega u=\theta .
$$

Also $\theta u=(\omega u) u=\omega u^{2}=\omega u=\theta$. Hence $\theta<u$.

4.5. Proposition. An idempotent is minimal if and only if it is contained in some minimal right ideal. 
Proof. Let $u$ be a minimal idempotent. Let $I$ be any minimal right ideal. Then by 4.4, $u I$ contains an idempotent $\theta$ such that $\theta<u$. Since $u$ is minimal, $u<\theta$. Thus

$$
u=u \theta \in u(u I)=u^{2} I=u I .
$$

Now by $4.2, u I$ is a minimal right ideal.

Conversely, let $I$ be a minimal right ideal and $u=u^{2} \in I$ be an idempotent. Let $\theta=\theta^{2} \in E$ be an idempotent such that $\theta<u$. Then

$$
(u \theta)(u \theta)=u(\theta u) \theta=u \theta^{2}=u \theta,
$$

so $u \theta$ is an idempotent. But $I$ is minimal so by $4.2, u \theta$ acts as a left identity on $I$. In particular

$$
u=(u \theta) u=u(\theta u)=u \theta
$$

and $u<\theta$ as desired.

4.6. Proposition. Let $I, M$ be minimal ideals in an $\mathcal{E}$-semigroup $E$, and $u \in M$ be an idempotent. Then there exists an idempotent $u^{\prime} \in I$ with $u \sim u^{\prime}$.

Proof. Note that $u I$ is a non-empty right ideal contained in $M$. Thus $u I=M$ and the set

$$
Q=\{p \in I \mid u p=u\}
$$

is a closed non-empty subsemigroup of $I$. By 1.18 there exists an idempotent $u^{\prime} \in Q$. Then $u u^{\prime}=u$ so $u<u^{\prime}$. On the other hand $u^{\prime} \in I$ so it's minimal by 4.5. It follows that $u \sim u^{\prime}$.

4.7. Lemma. Let $E$ be an $\mathcal{E}$-semigroup and $Q \subset E$ such that:

(1) $Q$ is closed and non-empty,

(2) $Q$ is a subsemigroup of $E$,

(3) $Q p \cap Q \neq \emptyset$ for $p \in E$, implies $p \in Q$,

(4) $Q$ is minimal with respect to (1), (2) and (3).

Then every idempotent $u \in Q$ is maximal in $E$.

Proof. Let $u \in Q$ be an idempotent, and assume that $v^{2}=v \in E$ with $u v=u$. Then

$$
u \in Q v \cap Q \neq \emptyset \quad \text { so } v \in Q
$$

Now

$$
\emptyset \neq L_{v}^{-1}(v) \cap Q=\{p \in Q \mid v p=v\} \equiv W \subset Q ;
$$

but $W$ satisfies (1), (2), and (3), so by minimality $Q=W$. In particular $v u=v$, so $u \sim v$.

The previous lemma motivates the following definition (see also $[\mathrm{AF}]$ ).

4.8. Definition. Let $E$ be an $\mathcal{E}$-semigroup. We refer to a subset $Q \subset E$ which satisfies conditions (1), (2), and (3) of 4.7, as a cancellation subsemigroup of $E$.

4.9. Example. Let $T$ be a group and $H$ be an infinite subgroup of $T$. Then $\beta H \backslash H \equiv \bar{H} \backslash H \subset \beta T \backslash T$ is a cancellation subsemigroup. 
Proof. Let $p \in \bar{H}$ and $t \in T$, and suppose that $p t \in \bar{H}$. Then $s t \in H$ for some $s \in H$. Since $H$ is a group this implies that $t \in H$. Now suppose that $q \in \beta T \backslash T$ with $p q \in \bar{H}$. Then there exists a net $t_{\alpha} \rightarrow q$ with $p t_{\alpha} \in \bar{H}$. The argument above implies that $t_{\alpha} \in H$ for all $\alpha$. Thus $q \in \bar{H} \backslash H$. This completes the proof.

We now state a proposition for maximal idempotents which is analogous to 4.5.

4.10. Proposition. Let $E$ be an $\mathcal{E}$-semigroup. Then $u \in J(E)$ is a maximal idempotent if and only if $u$ is contained in a minimal cancellation subsemigroup of E.

Proof. If $u \in Q$ for some minimal cancellation subsemigroup of $E$, then by $4.7 u$ is a maximal idempotent. Conversely assume that $u$ is maximal and set $W=\{p \in E \mid$ $u p=u\}=L_{u}^{-1}(u)$. Then $u \in W$ and $W$ is a closed subsemigroup of $E$. Now suppose that $q p \in W p \cap W$ with $q \in W$. Then $u=u(q p)=(u q) p=u p$, whence $p \in W$. This shows that $W$ is a cancellation subsemigroup of $E$. A straighforward Zorn's lemma argument shows that $W$ contains a minimal closed non-empty cancellation subsemigroup, say $Q$. Let $v \in Q \subset W$ be any idempotent. Then $u v=u$, so by the maximality of $u$ we have $v u=v$. Thus $v \in Q u \cap Q$ which implies that $u \in Q$ since $Q$ is cancellation. This completes the proof.

The following corollary is an immediate consequence of Propositions 4.5 and 4.10 .

4.11. Corollary. Let $c \in J(E)$. Then there are minimal and maximal idempotents $u$ and $m$ respectively, such that $u<c<m$.

Proof. Let $I$ be any minimal right ideal. By 4.4, $c I$ contains an idempotent $u$ such that $u<c$. Since $I$ is minimal, so is $c I$. Hence by $4.5 u$ is a minimal idempotent.

On the other hand $L_{c}^{-1}(c)$ is a cancellation subsemigroup of $E$. A Zorn's lemma argument produces a minimal cancellation subsemigroup $Q \subset L_{c}^{-1}(c)$. Now any idempotent $m \in Q$ is maximal by 4.10 , moreover $\mathrm{cm}=L_{c}(m)=c$ since $m \in$ $L_{c}^{-1}(c)$.

\subsection{Proposition. Let}

(1) $u=u^{2} \in E$ be an idempotent in $E$.

(2) $I \subseteq E$ be a closed subsemigroup of $E$ such that: $u I u \subset I$ and $v^{2}=v \in I$ with $v<u$ implies $u<v$.

(In other words no idempotent in $I$ is strictly less than u.) Then $u \in I$.

Proof. Notice that

$$
(u I)(u I) \subseteq(u I u) I \subseteq I I \subseteq I .
$$

Thus

$$
(u I)(u I)=\left(u^{2} I\right)(u I)=u(u I)(u I) \subseteq u I .
$$

Then $u I$ is also a closed subsemigroup, whence it contains an idempotent $p$. Let $p=u q$ with $q \in I$. Then

$$
u p=u(u q)=u^{2} q=u q=p .
$$

Now let $w=p u$. Then

$$
w^{2}=(p u)(p u)=p(u p) u=p^{2} u=p u=w=w u .
$$


Thus $w<u$, and since $w=p u=u q u \in u I u \subset I$, assumption (2) implies that $u<w$. Hence,

$$
u=u w=u(p u) \in u(u I) u=u I u \subseteq I .
$$

4.13. Remarks. (i) It follows immediately from 4.12 that if $I$ is a closed subsemigroup of an $\mathcal{E}$-semigroup $E$, and $u$ is a minimal idempotent with $u I u \subset I$, then $u \in I$.

(ii) If $I$ is a two-sided ideal in $E$, then $I$ contains every minimal idempotent of $E$. (This follows immediately from the previous remark since $u I u \subset I$ for every $u$.)

(iii) In [FK] (ii) above plays a crucial role in obtaining Ramsey-type theorems. We will use the stronger result (i) above to obtain multi-recurrence results in section 6 (see for example 6.3). Thus 4.12 extends the potential applicability of this algebraic technique.

\section{Notions of Recurrence}

Now we introduce various notions of recurrence. We shall see, however, that certain relationships which seem natural and intuitive, and are easily verified when $T$ is a group, fail to hold for general semigroups. In particular some of the notions of 'largeness' for subsets of $T$, and of recurrence for actions of $T$, do not satisfy the same relationships for a general semigroup that they do when $T$ is a group. One can define recurrent points either in terms of properties of elements of $\beta T$ that fix them, or through the 'largeness' of the set of their return times to a given neighborhood. We shall follow the latter approach and then prove results characterizing these notions in terms of the former. In connection with this characterization we would like to stress that the calculations are being carried out in the $\mathcal{E}$-semigroup $\beta T$, not in $\beta T \backslash T$. In particular when we refer to a minimal idempotent we are speaking of minimality in $\beta T$.

We begin by introducing and studying the relationships between various notions of largeness of subsets of $T$; then we turn to notions of recurrence.

5.1. Definitions. (i) Let $\mathcal{P}(T)$ be the collection of all subsets of $T$ and $\mathcal{A} \subseteq \mathcal{P}(T)$. Then the dual of $\mathcal{A}$ is defined by

$$
\mathcal{A}^{*}=\{B \subseteq T \mid A \cap B \neq \emptyset \text { for all } A \in \mathcal{A}\} .
$$

(ii) Let IP denote the class of IP-sets contained in $T$. A set $B$ is called an $I P^{*}$ set if it belongs to the class dual to IP.

(iii) The subset $A$ of $T$ is syndetic if there exists a finite subset $F$ of $T$ such that

$$
\bigcup\left\{R_{t}^{-1}(A) \mid t \in F\right\}=T \text {. }
$$

Let $\mathcal{S}$ denote the class of syndetic subsets of $T$.

When $T$ is a group, $R_{t}^{-1}(A)=A t^{-1}$. So that in this case $A$ is syndetic if and only if there exists a finite subset $F$ of $T$ with $A F=T$. Thus this definition reduces to the usual definition of a discretely syndetic subset.

(iv) The subset $A$ of $T$ is replete if for every finite subset $F$ of $T$ there exists $t \in T$ with $t F \subseteq A$. Let $\mathcal{R}$ denote the class of replete subsets of $T$.

When $T=\mathbb{Z}$, replete sets are usually called thick.

(v) The subset $A$ of $T$ is large if there is a finitely additive, positive, left-invariant measure $\mu$ on $T$ such that $\mu(A)>0$ and $\mu(T)=1$. 
When $T=\mathbb{Z}$, a subset of positive lower (Banach) density is large. In general, amenable groups admit a variety of finitely additive invariant measures with $\mu(T)=$ 1.

5.2. Remark. Although the definition of a syndetic set given above reduces to the usual one when $T$ is a group, in a general semigroup syndetic subsets can be 'small'. For example, suppose $T$ is a semigroup with the left identity structure. Then for any $t \in T$, and $A \subset T$,

$$
R_{t}^{-1}(A)= \begin{cases}T & \text { if } t \in A \\ \emptyset & \text { if } t \notin A\end{cases}
$$

so every non-empty subset is syndetic. On the other hand, in the semigroup with the right identity structure, for any $t \in T$, and $\emptyset \neq A \subset T, R_{t}^{-1}(A)=A$ so the only syndetic subset of $T$ is $T$ itself.

We shall see that in many of the following results, our usual intuition is correct if $\beta T \backslash T$ is invariant. In this case syndetic sets must at least be infinite.

5.3. Proposition. Let $A \subseteq T$ with $A \in \mathcal{S}$. Then $A t \in \mathcal{S}$ for all $t \in T$.

Proof. Let $t \in T$. Since $A \in \mathcal{S}$,

$$
T=\bigcup_{s \in F} R_{s}^{-1}(A)
$$

for some finite subset $F \subset T$. But $R_{s}^{-1}(A) \subset R_{s t}^{-1}(A t)$ so $T=\bigcup_{r \in F t} R_{r}^{-1}(A t)$, which implies that $A t$ is syndetic as desired.

5.4. Remark. The converse of 5.3 is false. For example, if $T$ is an infinite semigroup with zero structure and $A$ is any subset not containing 0 , then $A$ is not syndetic but $A t=\{0\}$ is syndetic for every $t \in T$.

5.5. Proposition. Let $A$ be a subset of the infinite semigroup, $T$. Then the following are equivalent:

(1) $A \in \mathcal{R}$,

(2) the family $\left\{R_{t}^{-1}(A) \mid t \in T\right\}$ has the finite intersection property,

(3) $A \in \mathcal{S}^{*}$.

Proof. (1) implies (2): Let $A \in \mathcal{R}$ and $F$ be a finite subset of $T$. Then there exists a $t \in T$ with $t F \subseteq A$. Hence $t \in \bigcap\left\{R_{s}^{-1}(A) \mid s \in F\right\}$.

(2) implies (3): Let $B \in \mathcal{S}$. Then there exists a finite subset $F$ of $T$ with $T=\bigcup\left\{R_{t}^{-1}(B) \mid t \in F\right\}$. By the assumption, there exists $s \in \bigcap\left\{R_{t}^{-1}(A) \mid t \in F\right\}$. Since there exists $t \in F$ with $s \in R_{t}^{-1}(B)$, i.e. st $\in A \cap B, A$ intersects every member of $\mathcal{S}$.

(3) implies (1): Let $A \in \mathcal{S}^{*}$ and $F$ be a finite subset of $T$. Let $A^{\prime}$ denote the complement of $A$ in $T$, and assume that $t F \cap A^{\prime} \neq \emptyset$ for all $t \in T$. Thus $\bigcup\left\{R_{s}^{-1}\left(A^{\prime}\right) \mid s \in F\right\}=T$. Hence $A^{\prime} \in \mathcal{S}$, which implies $A \cap A^{\prime} \neq \emptyset$. This contradiction completes the proof.

5.6. Corollary. For any infinite semigroup, the class $\mathcal{S}$ is dual to $\mathcal{R}$, i.e. $\mathcal{R}^{*}=\mathcal{S}$.

Proof. We have shown that $\mathcal{R}=\mathcal{S}^{*}$, hence $\mathcal{R}^{*}=\mathcal{S}^{* *} \supseteq \mathcal{S}$. Let $A \in \mathcal{R}^{*}$ and assume that $\bigcup\left\{R_{t}^{-1}(A) \mid t \in F\right\} \neq T$ for all finite subsets $F$ of $T$. Then $\left\{R_{t}^{-1}\left(A^{\prime}\right) \mid t \in T\right\}$ has the finite intersection property. Hence $A^{\prime} \in \mathcal{R}$. This contradicts the fact that $A \in \mathcal{R}^{*}$. 
5.7. Proposition. Let $T$ be an infinite semigroup and $A \subseteq T$ be a large subset. Then the set $\hat{A}=\left\{t \in T \mid A \cap L_{t}^{-1}(A) \neq \emptyset\right\}$ is an IP* set.

Proof. Let $\mu$ be a finitely additive left invariant measure on $T$ such that $\mu(A)>0$ and $\mu(T)=1$. Consider an IP-set $\pi(S)$, generated by the map $S: \mathbb{N} \rightarrow T$. Let $Z=\left\{z_{n}=S_{n} S_{n-1} \cdots S_{1} \mid n \in \mathbb{N}\right\} \subset \pi(S)$. If there exists $m>n$ with $L_{z_{m}}^{-1}(A)=L_{z_{n}}^{-1}(A)$, then

$$
0<\mu(A)=\mu\left(L_{z_{n}}^{-1}(A)\right)=\mu\left(L_{z_{n}}^{-1}(A) \cap L_{z_{m}}^{-1}(A)\right)=\mu\left(A \cap L_{S_{m} \cdots S_{n+1}}^{-1}(A)\right)
$$

and hence $S_{m} \cdots S_{n+1} \in \pi(S) \cap \hat{A}$. On the other hand even if the family of sets $\left\{L_{z_{n}}^{-1}(A) \mid n \in \mathbb{N}\right\}$ is distinct, the fact that each has (the same) positive measure and $\mu(T)=1$, implies that there exist $m>n$ with $\mu\left(L_{z_{n}}^{-1}(A) \cap L_{z_{m}}^{-1}(A)\right)>0$. The result then follows just as above.

We now define an action of $\beta T$ on the subsets of $\beta T$. This action allows us to give a characterization of the syndetic subsets of $T$ (see 5.11 and 5.16). We use this characterization in Proposition 5.12 to study replete subsets of $T$, and again in Proposition 5.21 to help identify an almost periodic point by looking at the elements of $\beta T$ which leave it fixed.

One way to define this action involves viewing the elements of $\beta T$ as ultrafilters on $T$. For a brief exposition of this approach see Appendix A, in particular the last remark. The following definition is equivalent but avoids making direct use of the language of ultrafilters.

5.8. Definition. Let $A \subset \beta T$ and $p \in \beta T$, we define

$$
A * p=L_{p}^{-1}(\bar{A}) .
$$

5.9. Lemma. Let $A \subset \beta T$ and $p, q \in \beta T$, then

$$
(A * p) * q=A *(p q) .
$$

Proof. $(A * p) * q=L_{q}^{-1}(\overline{A * p})=L_{q}^{-1}\left(\overline{L_{p}^{-1}(\bar{A})}\right)=L_{q}^{-1}\left(L_{p}^{-1}(\bar{A})\right)=L_{p q}^{-1}(\bar{A})=$ $A *(p q)$.

5.10. Remark. Notice that the semigroup operation on $\beta T$ defines another and in fact more natural action of $\beta T$ on subsets of $\beta T$ in the following way. Let $A \subset \beta T$ and $p \in \beta T$, then we denote by

$$
A p=\{a p \mid a \in A\} .
$$

Note that if $T$ is a group, $A=\{s\}, s \in T$ and $t \in T$, then $A * t=\left\{t^{-1} s\right\}$ whereas $A t=\{s t\}$.

5.11. Proposition. Let $T$ be an infinite semigroup, and $A \subseteq T$. Then the following statements are pairwise equivalent:

(1) $A \in \mathcal{S}$,

(2) $A * p \neq \emptyset$ for all $p \in \beta T$,

(3) $A * p \neq \emptyset$ for all minimal idempotents $p \in \beta T$.

Proof. (1) implies (2): Let $A \in \mathcal{S}$ and $p \in \beta T$. Then there exists a finite set $F$ with $\bigcup\left\{R_{t}^{-1}(A) \mid t \in F\right\}=T$. Hence

$$
p \in \bar{T}=\bigcup_{t \in F} \overline{R_{t}^{-1}(\bar{A})}=\bigcup_{t \in F} R_{t}^{-1}(\bar{A})
$$


and so there exists a $t \in F$ with $p t \in \bar{A}$. This implies that $t \in L_{p}^{-1}(\bar{A})=A * p$.

(2) implies (3): is clear.

(3) implies (1): Assume that $A * u \neq \emptyset$ for all minimal idempotents $u \in \beta T$. Assume that $\bigcup_{t \in F} R_{t}^{-1}(A) \neq T$ for all finite subsets $F \subset T$. Set

$$
B_{F}=\bigcap_{t \in F} R_{t}^{-1}(\overline{T \backslash A}) \subset \beta T .
$$

Then $\left\{B_{F} \mid F\right.$ a finite subset of $\left.T\right\}$ is a collection of closed subsets of $\beta T$ which has the finite intersection property. Since $\beta T$ is compact, there exists $p \in \beta T$ with $p t \in \overline{T \backslash A}=(\beta T) \backslash \bar{A}$ for all $t \in T$. Thus $\overline{p T} \subset(\beta T) \backslash \bar{A}$. Now $\overline{p T}$ is invariant, so it contains a minimal set $M ; M$ in turn is a subsemigroup of $\beta T$, so it contains a minimal idempotent $u$. It follows that $u t \in \beta T \backslash \bar{A}$ for all $t \in T$ and thus $\emptyset=$ $L_{u}^{-1}(\bar{A})=A * u$, a contradiction.

5.12. Proposition. Let $A \in \mathcal{R}$. Then for every $t \in T, R_{t}^{-1}(A)$ contains an $I P$ set.

Proof. Let $Q=\{p \in \beta T \mid T \subset A * p\}=\left\{p \in \beta T \mid T \subset L_{p}^{-1}(\bar{A})\right\}=\{p \in \beta T \mid$ $p t \in \bar{A}$ for all $t \in T\}=\left\{p \in \beta T \mid p \in R_{t}^{-1}(\bar{A})\right.$ for all $\left.t \in T\right\}$. The assumption implies that $\left\{R_{t}^{-1}(\bar{A}) \mid t \in T\right\}$ has the finite intersection property. Hence $Q$ is non-empty. We claim that $Q$ is $T$-invariant. Let $p \in Q$ and $t \in T$. Then for any $s \in T$, since $t s \in A * p,(p t) s=p(t s) \in \bar{A}$. Thus $T \subset A *(p t)$ and the claim is proved. Note that $Q$ is also closed.

Case (1). There exists $s \in T \cap Q$. Then $s T \subset \bar{A} \cap T=A$ which implies that $s T \subseteq R_{t}^{-1}(A)$ for all $t \in T$. Let $S: \mathbb{N} \rightarrow s T$ be any map. Then the IP-set $\pi(S)$ (see 3.1) generated by $S$ is contained in $s T$ and hence in $R_{t}^{-1}(A)$ for all $t \in T$.

Case (2). Suppose $Q \subset \beta T \backslash T$. The $T$-invariance of $Q$ implies that it is a closed subsemigroup and hence contains an idempotent $p$. Then for each $t \in T, p \in$ $R_{t}^{-1}(\bar{A})$. Since $R_{t}^{-1}(\bar{A})$ is a neighborhood of $p$, an application of Galvin's theorem completes the proof.

5.13. Proposition. Let $T$ be an infinite semigroup and $B \subset T$ be an $I P^{*}$ set. Then $B t \in \mathcal{R}^{*}=\mathcal{S}$ for all $t \in T$.

Proof. Let $A \in \mathcal{R}$ and $t \in T$. Since $R_{t}^{-1}(A)$ contains an IP-set, $B \cap R_{t}^{-1}(A) \neq \emptyset$. Thus $B t \cap A \neq \emptyset$.

5.14. Remark. (i) We should emphasize that the IP-set produced in 5.12 need not be infinite. If in the proof Case 2 holds, then Galvin's theorem does produce an infinite IP-set since $p \in \beta T \backslash T$. On the other hand, in Case 1, if $s T$ is finite, then any idempotent in $\overline{s T}=s T$ must be in $T$. Thus the condition: $s T$ is infinite for all $s \in T$ ensures that we can produce an infinite IP-set. For emphasis, we state this result as a corollary below. Notice that this condition follows if $T$ is infinite and satisfies the left cancellation law.

(ii) Notice that if $\beta T \backslash T$ is invariant and has the property: $s T$ is infinite for all $s \in T$, then all of the minimal idempotents in $\beta T$ are in $\beta T \backslash T$. To prove this, let $u \in T$ be a minimal idempotent of $\beta T$. Since $\overline{u T}$ is a minimal subset and by our assumption $\beta T \backslash T$ is invariant, $\overline{u T} \subset T$. Thus $u T$ is finite, which contradicts our assumption. This fact allows us to prove an analog of 5.12, which we also state below. 
5.15. Proposition. Let $A \in \mathcal{R}$ and assume that $s T$ is infinite for all $s \in T$. Then for every $t \in T, R_{t}^{-1}(A)$ contains an infinite IP-set.

Proof. See 5.12 and 5.14 .

5.16. Proposition. Let $T$ be an infinite semigroup, and $A \subseteq T$. Assume that $\beta T \backslash T$ is invariant and $s T$ is infinite for every $s \in T$. Then the following statements are pairwise equivalent:

(1) $A \in \mathcal{S}$,

(2) $A * p \neq \emptyset$ for all $p \in \beta T \backslash T$.

Proof. (1) implies (2) follows immediately from 5.11. On the other hand given the assumptions above, we saw in 5.14 that every minimal idempotent in $\beta T$ lies in $\beta T \backslash T$. Thus (2) implies (1) also follows from 5.11.

Now we begin the study of recurrence properties.

5.17. Notation. Let $(X, T)$ be a dynamical system, $x \in X$, and $W$ be a neighborhood of $x$. Let $A_{W}$ denote the set of return times of $x$ to $W$, i.e.

$$
A_{W}=\{t \in T \mid x t \in W\} .
$$

5.18. Lemma. The following are equivalent.

(1) For every neighborhood $W$ of $x$, the set $A_{W}$ is infinite.

(2) There exists $p \in \beta T \backslash T$ such that $x=x p$.

Proof. (1) implies (2): Assume that $A_{W}$ is infinite for every neighborhood $W$ of $x$. Consider the collection

$$
\left\{\overline{A_{W}} \backslash T \mid W \text { a neighborhood of } x\right\} \subset \beta T .
$$

Our assumption guarantees that this collection has the finite intersection property, so we can choose $p \in \bigcap \overline{A_{W}} \backslash T$. By 2.4 the map $\chi: \beta T \rightarrow X$ given by $\chi(q)=x q$ is continuous and therefore $x p=\chi(p) \in \chi\left(\overline{A_{W}}\right) \subset \bar{W}$ for all neighborhoods $W$ of $x$. This shows that $x p=x$.

(2) implies (1): Assume that $p \in \beta T \backslash T$ and $x p=x$. For any neighborhood $W$ of $x, \chi^{-1}(W)$ is a neighborhood of $p$ in $\beta T$. Thus by $2.2 \chi^{-1}(W)$ contains infinitely many elements of $T$, i.e. $A_{W}$ is infinite.

5.19. Definition. A point $x$ is recurrent if either of the two equivalent conditions in the lemma above holds.

5.20. Remark. If $\beta T \backslash T$ is a semigroup then the conditions above are equivalent to $x=x u$ for some idempotent $u \in \beta T \backslash T$. This is because in this case the set

$$
Q=\{p \in \beta T \backslash T \mid x p=x\}
$$

is a non-empty closed subsemigroup of $\beta T \backslash T$, and hence by 1.18, $Q$ contains an idempotent.

5.21. Proposition. Let $(X, T)$ be a dynamical system and $x \in X$. Then the following statements are equivalent

(1) For any neighborhood $W$ of $x$, the set $A_{W}$ is syndetic.

(2) $\overline{x T}$ is a minimal set containing $x$.

(3) Every minimal set $M \subset \beta T$ contains an idempotent $u$ such that $x u=x$. 
Proof. (1) implies (2) : Let $p \in \beta T$ and $W$ be any compact neighborhood of $x$. By the hypothesis $A_{W}$ is syndetic. Hence there exists a finite set $F \subset T$ such that $T=\bigcup_{s \in F} R_{s}^{-1}\left(A_{W}\right)$. Thus $p \in \overline{R_{s}^{-1}\left(A_{W}\right)}=R_{s}^{-1}\left(\overline{A_{W}}\right)$ for some $s \in F$. Now $x(p s) \in \bar{W}=W,(W$ is compact). This shows that $x p T \cap W \neq \emptyset$ for all $p \in \beta T$ and all neighborhoods $W$ of $x$. It follows that $x \in \overline{x p T} \subset \overline{x T}$ for all $p \in \beta T$. Thus $\overline{x T}=\overline{y T}$ for all $y \in \overline{x T}$, so $\overline{x T}$ is a minimal set containing $x$.

(2) implies (3) : Let $M$ be any minimal subset of $\beta T$. Then the minimality of $\overline{x T}$ implies that $\overline{x T}=x M$, and hence by our assumption $x \in x M$. Thus $L=\{p \in M \mid x p=x\}$ is non-empty. Notice that $M T \subset M$ implies that $M \beta T \subset M$. In particular $M$, and hence $L$, is a subsemigroup of $\beta T$. Therefore by 1.18 there exists a minimal idempotent $u \in L \subset M$ such that $x=x u$.

(3) implies (1) : Assume that (3) holds and let $W$ be any neighborhood of $x$. By 5.11 it is enough to show that $A_{W} * v \neq \emptyset$ for all minimal idempotents $v \in \beta T$. Let $v$ be any minimal idempotent, say $v \in M$ where $M$ is a minimal ideal in $\beta T$. Then by our assumption $x=x u$ for some minimal idempotent $u \in M$. Thus $u \in \overline{A_{W}}$, so $u \in L_{u}^{-1}\left(\overline{A_{W}}\right)=A_{W} * u=A_{W} *(v u)=\left(A_{W} * v\right) * u$, from which it follows that $A_{W} * v \neq \emptyset$.

5.22. Definition. Let $(X, T)$ be a dynamical system. A point $x \in X$ is uniformly recurrent or almost periodic if any of the three equivalent conditions in Proposition 5.21 hold.

5.23. Remark. (1) Condition (3) of 5.21 is equivalent to the statement that $x u=x$ for some minimal idempotent $u$. To see this recall that by 4.6 any minimal ideal contains an idempotent $u^{\prime}$ with $u \sim u^{\prime}$. Then $x=x u=x\left(u u^{\prime}\right)=x u^{\prime}$.

(2) If $\beta T \backslash T$ contains an invariant subset, (in particular this will be the case if $\beta T \backslash T$ is invariant) then $x$ is an almost periodic point if and only if $x u=x$ for some minimal idempotent in $\beta T \backslash T$.

(3) Thus when $\beta T \backslash T$ is invariant, uniformly recurrent points are recurrent. This can be seen directly as well because in such semigroups syndetic sets are infinite. However we have seen that for other semigroups this need not be true.

5.24. Lemma. Let $(X, T)$ be a dynamical system and $x, y \in X$. Then the following statements are equivalent.

(1) There exists a net $\left\{t_{\alpha}\right\} \subset T$ such that $\lim x t_{\alpha}=\lim y t_{\alpha}$.

(2) There exists an idempotent $u \in \beta T$ such that $x u=y u$.

(3) There exists a minimal idempotent $u \in \beta T$ such that $x u=y u$.

Proof. (1) implies (3). Assume that $\lim x t_{\alpha}=\lim y t_{\alpha}$ for some net $\left\{t_{\alpha}\right\} \subset T$. Since $\beta T$ is compact, by passing to a subnet if necessary, we may assume that $t_{\alpha} \rightarrow p \in \beta T$. Thus $x p=y p$ and

$$
Q=\{q \in \beta T \mid x q=y q\}
$$

is a non-empty closed right ideal of $\beta T$ and therefore contains a minimal right ideal and consequently a minimal idempotent $u$. (To see that $Q$ contains a minimal ideal, take any minimal ideal $I$ and consider $c I$ for $c \in Q$. Then $c I$ is a minimal ideal and $c I \subseteq Q$.) The implications : (3) implies (2) and (2) implies (1) are immediate.

5.25. Definition. (1) If any of the three equivalent conditions in the lemma above hold, then the points $x$ and $y$ are said to be proximal. 
(2) A point $x \in X$ is a distal point if it is not proximal to any point in its orbit closure other than itself.

5.26. Remark. The definition above corresponds to the usual notions of proximal and distal when $T$ is a group. It should be noted however that in a general semigroup the elements need not act as one-one maps, indeed it may happen that $x t=y t$ but $x \neq y$. In this case $x$ is proximal to $y$ according to Definition 5.25.

5.27. Proposition. Let $(X, T)$ be a dynamical system and $x \in X$. Then the following statements are equivalent:

(1) $x$ is a distal point.

(2) $x u=x$ for all minimal idempotents $u \in \beta T$.

(3) $x u=x$ for all idempotents $u \in \beta T$.

Proof. (1) implies (2) : Let $u$ be any minimal idempotent in $\beta T$. Then $x$ and $x u$ are proximal, and $x u \in \overline{x T}$. Hence if $x$ is a distal point, it follows that $x=x u$.

(2) implies (3) : Let $u \in \beta T$ be any idempotent. Then by 4.11 there exists a minimal idempotent $v<u$. Assuming (2) it follows that $x u=(x v) u=x(v u)=$ $x v=x$.

(3) implies (1) : Assume that $x u=x$ for all idempotents $u$. Let $y \in \overline{x T}$ be proximal to $x$, say $x r=y r$ for some $r \in \beta T$. Then $x q=y q$ for all $q$ in the right ideal $r(\beta T)$. Choose a minimal right ideal $M \subset r(\beta T)$. Since $\overline{x T}$ is a minimal set, $\overline{x T}=\overline{y T}$ and $y$ is an almost periodic point. By 5.21 there exists an idempotent $u \in M$ with $y=y u$. Then $y=y u=x u=x$. This proves that $x$ is a distal point.

5.28. Corollary. Let $(X, T)$ be a dynamical system and $x \in X$ be a distal point. Then for any neighborhood $W$ of $x$, the set $A_{W}$ is an $I P^{*}$ set.

Proof. Let $W$ be any neighborhood of $x$, and $\pi(S)$ be the IP-set generated by $S$. Then by Theorem 3.3, $\overline{\pi(S)}$ contains an idempotent $u$. Since $x$ is distal, it follows from Proposition 5.27 that $x u=x$. Thus $u \in \overline{A_{W}} \cap \overline{\pi(S)}$ which implies that $A_{W} \cap \pi(S) \neq \emptyset$ (see Remark 2.2). This shows that $A_{W}$ is an IP* set.

5.29. Remark. (1) As in Remark 5.23 condition (2) of 5.27 is equivalent to the statement that $x u=x$ for all idempotents in some minimal ideal. Hence if $\beta T \backslash T$ contains an invariant set, (in particular if $\beta T \backslash T$ is invariant), then $x$ is a distal point if and only if it is fixed by all the idempotents in $\beta T \backslash T$.

(2) Corollary 5.28 is a generalization of one implication of a characterization due to Furstenberg, of a distal point in terms of its set of return times. In the following corollary we show that the converse also holds when $\beta T \backslash T$ contains an invariant set.

5.30. Corollary. Let $T$ be an infinite semigroup such that $\beta T \backslash T$ contains an invariant set, $(X, T)$ a dynamical system and $x \in X$. Then $x$ is a distal point if and only if for any neighborhood $W$ of $x$, the set $A_{W}$ is an IP* set.

Proof. Necessity is just 5.28. Conversely, to show that $x$ is a distal point, by Remark 5.29 it is enough to show that $x u=x$ for every idempotent $u \in \beta T \backslash T$. Let $u \in \beta T \backslash T$ be an idempotent and suppose that $x u \neq x$. Then there exists a neighborhood $W$ of $x$ such that $x u \notin W$, (since $X$ is compact Hausdorff and hence locally compact, we may assume without loss of generality that $W$ is compact). 
Thus $u \notin \overline{A_{W}}$ and hence $u \in \overline{T \backslash A_{W}}$ by Remark 2.2. Applying 3.3 (Galvin's theorem) we conclude that $T \backslash A_{W}$ contains an IP set. This contradicts the assumption that $A_{W}$ is an IP* set. Therefore $x u=x$ and $x$ is a distal point.

5.31. Definition. A point $x \in X$ is product recurrent if given any other dynamical system $(Y, T)$ and a recurrent point $y \in Y$, the point $(x, y)$ is a recurrent point of the product dynamical system $X \times Y$.

5.32. Proposition. Assume

(1) $(X, T)$ is a flow,

(2) $x \in X$,

(3) $\beta T \backslash T$ is a semigroup.

Then the following statements are equivalent:

(a) $x$ is product recurrent.

(b) $x u=x$ for every maximal idempotent $u \in \beta T \backslash T$.

Proof. (a) implies (b) : (Note that this implication does not require assumption (3).) Assume that $x$ is product recurrent. Let $u \in \beta T \backslash T$ be a maximal idempotent. Then $u$ is a recurrent point for the dynamical system $(\beta T, T)$. Hence there exists an idempotent $v \in \beta T \backslash T$ with $(x, u) v=(x, u) \in X \times \beta T$. Thus, $u v=u$. The maximality of $u$ implies $u \sim v$, so $x u=(x v) u=x(v u)=x v=x$.

(b) implies (a) : Assume that $x u=x$ for every maximal idempotent $u \in \beta T \backslash T$. Let $y \in Y$ be a recurrent point for the dynamical system $(Y, T)$. Then since $\beta T \backslash T$ is a semigroup, there exists an idempotent $v \in \beta T \backslash T$ with $y v=y$ (see Remark 5.20). Let $w$ be a maximal idempotent in $\beta T \backslash T$ such that $v<w$. Then,

$$
(x, y) w=(x w, y w)=(x, y v w)=(x, y v)=(x, y) \in X \times Y .
$$

Thus $x$ is product recurrent.

The fact that when $\beta T \backslash T$ is a semigroup, every distal point is a product recurrent point is an now an immediate consequence of Proposition 5.27 and Proposition 5.32. We proceed to give conditions under which the two notions coincide. In particular we will see that this is the case when $T$ is a group. Our approach relies on the following proposition which is of independent interest.

\subsection{Proposition. Assume}

(1) $T$ is a countably infinite semigroup,

(2) $\beta T \backslash T$ is invariant,

(3) the left cancellation law holds in $T$.

Then the maximal idempotents in the $\mathcal{E}$-semigroup $\beta T \backslash T$ are dense in $J(\beta T \backslash T)$.

Proof. The proof relies on a modification of the argument used in the proof of Galvin's theorem (3.3). This modification is a generalization of the 'rarification procedure' introduced in $\mathrm{AF}$.

Let $u=u^{2} \in \beta T \backslash T$ be an idempotent and $V \subset T$ with $u \in \bar{V}$. We will show that $V$ contains an IP-set $\pi(S)$ with the following additional property:

If $g \in T \backslash \pi\left(S^{j}\right)$ and $g$ is not a left identity of $T$, then there exists $n \in \mathbb{N}$ such that $\pi\left(S^{n}\right) g \cap \pi\left(S^{j}\right)=\emptyset$.

This additional property guarantees that the asymptotic subsemigroup

$$
\pi_{\infty}(S)=\bigcap\left\{\overline{\pi\left(S^{n}\right)} \mid n \in \mathbb{N}\right\} \cap(\beta T \backslash T) \subset \bar{V}
$$


has the cancellation property, and so completes the proof since by $4.7, \pi_{\infty}(S)$ then contains a maximal idempotent.

Before going further it is worth mentioning two simple consequences of our assumptions. First note that assumption (3) implies that any right-identity $r \in T$ is also a left identity, indeed:

$$
y(r x)=(y r) x=y x \quad \text { and hence } \quad r x=x
$$

for all $x, y \in T$. The second consequence, this time of assumption (2), is that the set of left-identities in $T$ (which we will denote by $L$ ) is finite: if $f \in L$, then $L \subset L_{f}^{-1}(f)$ and the latter is finite by 2.11 .

To see that $\pi_{\infty}(S)$ is a cancellation subsemigroup (see Definition 4.8) of $\beta T \backslash T$, let $p \in \pi_{\infty}(S)$ and $q \in(\beta T \backslash T) \backslash \pi_{\infty}(S)$. Then there exists a net $\left(g_{\alpha}\right) \subset T \backslash \pi\left(S^{j}\right)$ with $g_{\alpha} \rightarrow q$. Since the set of left identities in $T$ is finite we may assume that none of the $g_{\alpha}$ are left identities (otherwise $q \in T$ ). Now applying the property above to $g_{\alpha}$, there exists $n \in \mathbb{N}$ such that $\pi\left(S^{n}\right) g_{\alpha} \cap \pi\left(S^{j}\right)=\emptyset$. But $p \in \pi_{\infty}(S)$ so there exist $\left\{r_{\beta}\right\} \subset \pi\left(S^{n}\right)$ such that $r_{\beta} \rightarrow p$. Thus $p g_{\alpha}=\lim _{\beta} r_{\beta} g_{\alpha} \in \overline{T \backslash \pi\left(S^{j}\right)}$. It follows that $p q=\lim _{\alpha} p g_{\alpha} \notin \overline{\pi\left(S^{j}\right)}$ and hence $p q \notin \pi_{\infty}(S)$.

We now construct an IP-set $\pi(S)$ which satisfies the desired property. First, order the elements of $T$, say $T=\left\{g_{1}, g_{2}, \cdots\right\}$; this order will remain fixed throughout the proof.

Now $L_{u}(u)=u^{2} \in \bar{V}$ so $u \in \bar{V} \cap L_{u}^{-1}(\bar{V})$. Since the latter is both open and closed in $\beta T$, and $u \notin T$, we can begin by choosing $S_{1} \in V \cap L_{u}^{-1}(\bar{V})$. In order to choose an appropriate $S_{2}$ we set

$$
\begin{gathered}
L=\{e \mid e \text { is a left identity of } T\}, \\
Q_{1}=\left\{S_{1}\right\}, \\
A_{1}=\bigcup\left\{R_{t g_{1}}^{-1}(s) \mid t, s \in Q_{1}\right\}, \\
B_{1}=\bigcup\left\{R_{t}^{-1}\left(s g_{1}\right) \mid t, s \in Q_{1}\right\}, \\
C_{1}=\bigcup\left\{R_{t g_{1}}^{-1}(L) \mid t \in Q_{1}\right\} \cup R_{g_{1}}^{-1}(L), \\
R_{1}=\left\{S_{1}\right\} \cup A_{1} \cup B_{1} \cup C_{1} \cup L .
\end{gathered}
$$

Let $V_{1}=V \backslash R_{1}$. Notice that it follows from assumption (2) and 2.11 that each of the sets above (in particular $R_{1}$ ) is finite. Thus, since $u \notin T, L_{u}(u)=u \in \overline{V_{1}}$ and hence $u \in \overline{V_{1}} \cap L_{u}^{-1}\left(\overline{V_{1}}\right)$. Using 3.5 we choose $S_{2} \in T$ so that

$$
S_{2} \in V_{1} \cap L_{u}^{-1}\left(\overline{V_{1}}\right)
$$

and

$$
S_{2} S_{1} \in V \cap L_{u}^{-1}(\bar{V}) .
$$

Note that $S_{1}, S_{2}$ are distinct, $S_{2} \in V \backslash R_{1}$, and $S_{1}, S_{2}, S_{2} S_{1} \in V \cap L^{-1}(\bar{V})$.

In order to choose an appropriate $S_{3}$ we set

$$
\begin{gathered}
Q_{2}=\left\{S_{1}, S_{2}, S_{2} S_{1}\right\}, \\
A_{2}=\bigcup\left\{R_{t g_{m}}^{-1}(s) \mid t, s \in Q_{2}, 1 \leq m \leq 2\right\},
\end{gathered}
$$




$$
\begin{gathered}
B_{2}=\bigcup\left\{R_{t}^{-1}\left(s g_{m}\right) \mid t, s \in Q_{2}, 1 \leq m \leq 2\right\}, \\
C_{2}=\bigcup\left\{R_{t_{m}}^{-1}(L) \mid t \in Q_{2}, 1 \leq m \leq 2\right\} \cup\left\{R_{g_{m}}^{-1}(L) \mid 1 \leq m \leq 2\right\}, \\
R_{2}=\left\{S_{1}, S_{2}\right\} \cup A_{2} \cup B_{2} \cup C_{2} \cup L .
\end{gathered}
$$

Let $V_{2}=V \backslash R_{2}$. Again it follows from assumption (2) and 2.11 that each of the sets above (in particular $R_{2}$ ) is finite. Thus, since $u \notin T, L_{u}(u)=u \in \overline{V_{2}}$ and hence $u \in \overline{V_{2}} \cap L_{u}^{-1}\left(\overline{V_{2}}\right)$. Using 3.5 we choose $S_{3} \in T$ so that

$$
S_{3} \in V_{2} \cap L_{u}^{-1}\left(\overline{V_{2}}\right)
$$

and

$$
\left\{S_{3} S_{1}, S_{3} S_{2}, S_{3} S_{2} S_{1}\right\} \subset V \cap L_{u}^{-1}(\bar{V}) .
$$

Note that $S_{1}, S_{2}, S_{3}$ are distinct, $S_{3} \in V \backslash R_{2}$, and

$$
\left\{S_{1}, S_{2}, S_{3}, S_{2} S_{1}, S_{3} S_{1}, S_{3} S_{2}, S_{3} S_{2} S_{1}\right\} \subset V \cap L^{-1}(\bar{V}) .
$$

Continuing in this way, having defined a one-one map $S:\{1,2, \ldots, k\} \rightarrow T$ such that $S_{i+1} \in V \backslash R_{i}$ for $1 \leq i \leq k-1$, and

$$
S_{F} \in V \cap L^{-1}(\bar{V}) \text { for all } F=\left\{i_{1}<i_{2}<\cdots<i_{m}\right\} \subset\{1,2, \ldots, k\},
$$

in order to choose an appropriate $S_{k+1}$ we set

$$
\begin{gathered}
Q_{k}=\left\{S_{F} \mid F \subset\{1, \ldots, k\}\right\}, \\
A_{k}=\bigcup\left\{R_{t g_{m}}^{-1}(s) \mid t, s \in Q_{k}, 1 \leq m \leq k\right\}, \\
B_{k}=\bigcup\left\{R_{t}^{-1}\left(s g_{m}\right) \mid t, s \in Q_{k}, 1 \leq m \leq k\right\}, \\
C_{k}=\bigcup\left\{R_{t g_{m}}^{-1}(L) \mid t \in Q_{k}, 1 \leq m \leq k\right\} \cup\left\{R_{g_{m}}^{-1}(L) \mid 1 \leq m \leq k\right\}, \\
R_{k}=\left\{S_{1}, S_{2}, \ldots, S_{k}\right\} \cup A_{k} \cup B_{k} \cup C_{k} \cup L .
\end{gathered}
$$

Let $V_{k}=V \backslash R_{k}$. Again it follows from assumption (2) and 2.11 that each of the sets above (in particular $R_{k}$ ) is finite. Thus as before $u \in \overline{V_{k}} \cap L_{u}^{-1}\left(\overline{V_{k}}\right)$. Using 3.5 we choose $S_{k+1} \in T$ so that

$$
S_{k+1} \in V_{k} \cap L_{u}^{-1}\left(\overline{V_{k}}\right)
$$

and

$$
\left\{S_{k+1} S_{F} \mid F \subset\{1,2, \ldots, k\}\right\} \subset V \cap L_{u}^{-1}(\bar{V}) .
$$

Note that $S_{1}, S_{2}, \ldots, S_{k+1}$ are distinct, $S_{k+1} \in V \backslash R_{k}$, and $\left\{S_{F} \mid F \subset\{1,2, \ldots, k+\right.$ $1\}\} \subset V \cap L^{-1}(\bar{V})$.

Induction on $k$ now guarantees the existence of a one-one map $S: \mathbb{N} \rightarrow T$ such that $S_{k+1} \in V \backslash R_{k}$ for all $k \in \mathbb{N}$ and such that $S_{F} \in V \cap L_{u}^{-1}(\bar{V})$ for all $F=\left\{i_{1}<i_{2}<\cdots<i_{m}\right\} \subset \mathbb{N}$.

Now let $g \in T$, say $g=g_{n-1}$ for some $n \in \mathbb{N}$. Assume that $g$ is not a left identity, $g \notin \pi\left(S^{j}\right)$, and $\pi\left(S^{n}\right) g \cap \pi\left(S^{j}\right) \neq \emptyset$. (We will argue toward a contradiction.) Then there exist $n \leq a_{1}<a_{2}<\cdots<a_{k}$ and $j \leq b_{1}<b_{2}<\cdots<b_{m}$ such that

$$
S_{a_{k}} S_{a_{k-1}} \cdots S_{a_{1}} g=S_{b_{m}} S_{b_{m-1}} \cdots S_{b_{1}} \text {. }
$$


Assume that $a_{k}>b_{m}$, then $S_{a_{k}} \in R_{s}^{-1}\left(S_{b_{m}} S_{b_{m-1}} \cdots S_{b_{1}}\right)$, where $s=S_{a_{k-1}} \cdots S_{a_{1}} g$. Since $n-1<a_{k}, S_{a_{k}} \in A_{a_{k}} \subseteq R_{a_{k}}$. This is in contradiction to our choice of $S_{a_{k}}$. On the other hand if $a_{k}<b_{m}$ then $S_{b_{m}} \in R_{s}^{-1}\left(S_{a_{k}} S_{a_{k-1}} \cdots S_{a_{1}} g\right)$ where $s=S_{b_{m-1}} \cdots S_{b_{1}}$. Thus $S_{b_{m}} \in B_{b_{m}} \subseteq R_{b_{m}}$, again a contradiction. Hence $a_{k}=$ $b_{m}$. Now applying the left cancellation law and continuing this way, we end up with the following two possibilities. For some $r$, either $g=S_{b_{r}} S_{b_{r-1}} \cdots S_{b_{1}}$ or $S_{a_{r}} S_{a_{r-1}} \cdots S_{a_{1}} g=S_{a_{r}}$.

In the first case $g=S_{b_{r}} S_{b_{r-1}} \cdots S_{b_{1}} \in \pi\left(S^{j}\right)$-a contradiction. In the second case $S_{a_{r}} S_{a_{r-1}} \cdots S_{a_{1}} g x=S_{a_{r}} x$ for all $x \in T$. Hence the left cancellation property implies that either $g \in L$ or $S_{a_{r-1}} \cdots S_{a_{1}} g \in L$ depending on whether $r=1$ or $r>1$. Since we have assumed that $g \notin L, r$ must be greater than 1 .

If $r=2$, then $S_{a_{1}} g \in L$, and if $r>2$, then $S_{a_{r-1}} \in R_{s}^{-1}(L)$, where $s=$ $S_{a_{r-2}} \cdots S_{a_{1}} g$. In either case, since $g \equiv g_{n-1}$, we have $S_{a_{r-1}} \in C_{n-1} \subset R_{n-1}$. But $R_{n-1} \subset R_{a_{r-1}}$ because $n \leq a_{1} \leq a_{r-1}$. This again is in contradiction to our choice of $S_{a_{r-1}}$. It follows that $\pi(S)$ has the desired property, which completes the proof.

5.34. Corollary. Let $T$ be a group. Then the maximal idempotents in $\beta T \backslash T$ are dense in $J(\beta T \backslash T)$.

Proof. Let $u \in J(\beta T \backslash T)$ be any idempotent and $V \subset \beta T$ be any neighborhood of $u$. Then by 3.7 there exists a countable subgroup $H$ of $T$ and an idempotent $v \in V \cap(\bar{H} \backslash H)$. Applying 5.33 to $\bar{H} \backslash H$, we obtain an idempotent $m \in V$ which is maximal in $\bar{H} \backslash H$. We claim that $m$ is also maximal in $\beta T \backslash T$. This follows from the fact that $\bar{H} \backslash H$ is a cancellation subsemigroup of $\beta T \backslash T$ (which was proven in 4.9). To see this suppose $w \in J(\beta T \backslash T)$ with $m<w$. Then $m w=m$, so $(\bar{H} \backslash H) w \cap(\bar{H} \backslash H) \neq \emptyset$, which implies that $w \in \bar{H} \backslash H$. Since $m$ is maximal in $\bar{H} \backslash H$, it follows that $m \sim w$. This show that $m$ is maximal in $\beta T \backslash T$, which completes the proof.

\subsection{Proposition. Assume}

(1) $(X, T)$ is a flow,

(2) $x \in X$,

(3) $T$ is countably infinite,

(4) $\beta T \backslash T$ is invariant,

(5) the left cancellation law holds in $T$.

Then the following statements are equivalent:

(a) $x$ is a distal point.

(b) $x$ is product recurrent.

Proof. Note that the assumption that $\beta T \backslash T$ is invariant guarantees that $\beta T \backslash T$ is a semigroup. Thus Proposition 5.32 applies. As we remarked earlier the fact that (a) implies (b) is an immediate consequence of Propositions 5.27 and 5.32.

(b) implies (a) : Assume that $x$ is product recurrent. Then by Proposition 5.32, $x u=x$ for every maximal idempotent $u \in \beta T \backslash T$. Let $v$ be any idempotent in $\beta T \backslash T$. Then by 5.33 , there exists a net $u_{\alpha}$ of maximal idempotents in $\beta T \backslash T$ such that $u_{\alpha} \rightarrow v$. Thus for any $x \in X$,

$$
x v=\lim x u_{\alpha}=x .
$$

The fact that $x$ is a distal point now follows from Remark 5.29 and Proposition 5.27 . 
5.36. Corollary. Assume

(1) $(X, T)$ is a flow,

(2) $x \in X$,

(3) $T$ is an infinite group.

Then the following statements are equivalent:

(a) $x$ is a distal point.

(b) $x$ is product recurrent.

Proof. Note that the assumption that $T$ is a group guarantees that $\beta T \backslash T$ is invariant and hence is a semigroup. Thus Proposition 5.32 applies and the fact that (a) implies (b) is an immediate consequence of Propositions 5.27 and 5.32.

(b) implies (a) : Assume that $x$ is product recurrent. Then by Proposition 5.32, $x u=x$ for every maximal idempotent $u \in \beta T \backslash T$. Let $v$ be any idempotent in $\beta T \backslash T$. Then by Corollary 5.34, there exists a net $u_{\alpha}$ of maximal idempotents in $\beta T \backslash T$ such that $u_{\alpha} \rightarrow v$. Thus for any $x \in X$,

$$
x v=\lim x u_{\alpha}=x .
$$

The fact that $x$ is a distal point now follows from Remark 5.29 and Proposition 5.27 .

\section{Abstract Multiple Birkhoff Recurrence}

Now we turn to multiple recurrence. Many interesting multi-recurrence results can be proved by locating recurrent points in appropriate dynamical systems. First, we shall describe the problem of locating recurrent points more precisely. Let $X$ be a compact Hausdorff space acted upon by two semi-groups $T$ and $S$ such that the $T$ and $S$ actions commute (i.e. $t s=s t, t \in T, s \in S$, where we view $S$ and $T$ as subsets of $\left.X^{X}\right)$. Let $Y$ be a closed $T$-invariant subset of $X$ such that the flow $(Y, T)$ has certain recurrence properties (for example minimality). The problem then is: What can be said about the set $R=\{y \in Y \mid y$ is $S$ recurrent $\}$ ? Notice that $Y$ need not be $S$ invariant. When $Y$ is metrizable, the goal is to show that $R$ is a residual subset of $Y$.

In the original proof of multiple Birkhoff recurrence (the so-called MBR theorem; see Theorem 2.6 of [F]) Furstenberg first proved a result about locating recurrent points in a "homogenous set" (Proposition 2.4 of [F]) and then used this result along with a certain induction argument to prove the multi-recurrence theorem. This approach to multi-recurrence will be discussed (and generalized) in Appendix B.

The second (and entirely different) proof of multi-recurrence result appears in the works of [FK] and that uses the enveloping semigroup approach. (To be precise, in [FK] Furstenberg and Katznelson prove the van der Waerden's theorem and not the MBR theorem itself.) In this section we take yet another algebraic approach. We give a different set of conditions which do not involve any direct assumption about how $S$ acts on $Y$. Instead the key condition (see 6.1 (4) below) involves the relationship between the idempotents in $\bar{T}$ and $\overline{S T}$. This approach also allows us to give direct proofs of more general multi-recurrence results (see 6.10 and 6.12). We shall deduce (see Remark 6.11) the "classical" multi-recurrence theorem (by "classical" we mean the multiple recurrence theorem for powers of a single continuous transformation - to be precise - Theorem 2.1 of [F1]) from 6.10 and the the one for 
commuting transformations (Theorem 2.6 of $[\mathrm{F}]$ ) from 6.12. Finally, we recall (iii) of Remark 4.13 which points out the advantage of our approach over that of [FK].)

Unless specified to the contrary the following will be in force throughout this section.

6.1. Standing assumptions. (1) Let $S$ and $T$ be infinite semigroups.

(2) Let $X$ be a compact Hausdorff space on which both $S$ and $T$ act continuously (on the right). That is $(X, T)$ and $(X, S)$ are both dynamical systems. We shall view $S$ and $T$ as subsets of $X^{X}$.

(3) Viewed as elements of $X^{X}$, st $=t s$, for all $s \in S, t \in T$.

(4) If $u$ is a minimal idempotent in $\bar{T}$ and $p$ is an idempotent in $\overline{S T}$ with $p<u$, then $u<p$, (i.e. no idempotent in $\overline{S T}$ is strictly smaller than $u$ ). Note that all closures are taken in $X^{X}$.

6.2. Lemma. Let $u$ be a minimal idempotent in $\bar{T}$. Then,

(1) $u \in \overline{S T}$ and

(2) $(u, u)$ is a minimal idempotent in $\bar{T} \times \overline{S T}$.

Proof. (1) Notice that $t S T \subset S T, t S T r \subset S T(t, r \in T)$. Hence $u \overline{S T} u \subset \overline{S T}$, and so $u \in \overline{S T}$ by (4) of 6.1 and 4.12 .

(2) Now let $(v, \omega)$ be an idempotent in $\bar{T} \times \overline{S T}$ with $(v, \omega)<(u, u)$. Then $v<u, \omega<u$, whence $u<v$ since $u$ is minimal in $\bar{T}$ and $u<\omega$ by (4) of 6.1. Thus $(u, u)<(v, \omega)$.

6.3. Abstract Multi-Recurrence Lemma. Let

(1) $\mathcal{H}$ be a collection of subsets of $S$ such that

(a) $\mathcal{H}$ has the finite intersection property,

(b) given $H \in \mathcal{H}$ and $s \in H$ there exists $K \in \mathcal{H}$ with $K s \subset H$,

(2) $u$ be a minimal idempotent in $\bar{T} \subset X^{X}$,

(3) $U$ be a neighborhood of $u$ in $\bar{T}$,

(4) $H \in \mathcal{H}$.

Then there exist $t \in T$, and $s \in H$ with $t, t s \in U$.

Proof. For every $H \in \mathcal{H}$ let $H^{*}=\{(t, t s) \mid t \in T, s \in H\}$, and $\overline{H^{*}}$ be the closure of $H^{*}$ in $X^{X} \times X^{X}$. By (1)(a) the family $\left\{\overline{H^{*}} \mid H \in \mathcal{H}\right\}$ has the finite intersection property. Thus $I=\bigcap\left\{\overline{H^{*}} \mid H \in \mathcal{H}\right\}$ is closed and non-empty. We now show that $I$ is a subsemigroup of $\bar{T} \times \overline{S T}$.

Let $H \in \mathcal{H}, t \in T$ and $s \in H$. Choose $K \in \mathcal{H}$ with $K s \subset H$. If $(r, r k) \in K^{*}$, then $(r, r k)(t, t s)=(r t, r t k s) \in H^{*}$. Thus $K^{*}(t, t s) \subset H^{*}$, whence $I(t, t s) \subset \overline{K^{*}}(t, t s) \subset$ $\overline{H^{*}}$. This implies that $I(t, t s) \subset \overline{H^{*}}$ for all $t \in T$ and $s \in H$. Hence $I H^{*} \subset \overline{H^{*}}$. Thus, $I^{2} \subset I \overline{H^{*}} \subset \overline{H^{*}}(H \in \mathcal{H})$, and consequently $I^{2} \subset I$.

Now observe that $(r, r) H^{*}(t, t) \subset H^{*}(r, t \in T)$ implies that $(u, u) I(u, u) \subset I$. Hence $(u, u) \in I$ by $(2)$ of 6.12 and 4.12 . But $U \times U$ is a neighborhood of $(u, u)$, so $\emptyset \neq(U \times U) \cap I \subset(U \times U) \cap \overline{H^{*}}$ which implies that $(U \times U) \cap H^{*} \neq \emptyset$.

\subsection{Proposition. Let}

(1) $p^{2}=p \in \beta S \backslash S$,

(2) $V$ be a neighborhood of $p$ in $\beta S$,

(3) $Y$ be a closed, metrizable, $T$-invariant subset of $X$ such that the almost periodic points of $(Y, T)$ are dense in $Y$.

Then $Q=\left\{y \in Y \mid y q=y\right.$ for some $\left.q^{2}=q \in V\right\}$ is a residual subset of $Y$. 
Proof. Let $W$ be a closed neighborhood of $p$ in $\beta S$ with $W \subset V$. There exists $H: \mathbb{N} \rightarrow S$ such that $\pi\left(H^{n}\right) \subset W$ for all $n \in \mathbb{N}$. Let

$$
Z_{n}=\left\{y \in Y \mid d(y, y s)<\frac{1}{n} \text { for some } s \in \pi\left(H^{n}\right)\right\} .
$$

Then $Z_{n}$ is clearly an open subset of $Y$. We will show that it is dense in $Y$.

First recall that the family $\left\{\pi\left(H^{m}\right) \mid m \in \mathbb{N}\right\}$ satisfies (1) of 6.3. Now let $U$ be a non-empty open subset of $Y$. Then there exists an open subset $N$ of $X$ such that $N \cap Y$ is a non-empty subset of $Y$ of diameter $1 / n$ which is contained in $U$. By (3) there exists an almost periodic point $z$ of $(X, T)$ with $z \in N \cap Y$, whence $z u=z$ for some minimal idempotent $u$ in $\bar{T}$.

Now $L=\{r \in \beta T \mid z r \in N\}$ is a neighborhood of $u$ whence by 6.3 there exist $t \in T, s \in \pi\left(H^{n}\right)$ with $t, t s \in L$. Since $Y$ is invariant under $T$, this implies that $z t \in N \cap Y$. Consequently $z t \in U \cap Z_{n}$.

Finally let $Z=\bigcap\left\{Z_{n} \mid n \in \mathbb{N}\right\}$, and $z \in Z$. Since $z \in Z_{n}$, there exists $s_{n} \in \pi\left(H^{n}\right)$ with $d\left(z, z s_{n}\right)<1 / n$. Let $r$ be a limit point of $\left\{s_{n}\right\}$ in $\beta S$. Then $z r=z$ and $r \in \pi_{\infty}(H)$. Since $\pi_{\infty}(H)$ is a closed non-empty semigroup, there exists an idempotent $q \in \pi_{\infty}(H)$ with $z q=z$. Thus $z \in Q$ since $\pi_{\infty}(H) \subset W \subset V$.

6.5. Remarks. (1) The assumption that the almost periodic points are dense in $Y$ cannot be eliminated. To see this let $T$ be a countable group and $Y$ be the onepoint compactification of $T$. Then $y p=\omega$ for every $y \in Y$ and $p \in \beta T \backslash T$, where $\omega$ denotes the point at infinity in $Y$. Hence in this case $Q=\{\omega\}$, in particular $Q$ is not residual in $Y$.

(2) Notice that $Y$ need not be invariant under $S$.

(3) In general the set $Q$ of Proposition 6.4 need not consist of recurrent points because there may be idempotents $q \in S$. If we assume that $S$ has only finitely many idempotents, (such as when $S$ is a group or subsemigroup of a group) then we may as well assume that the neighborhood $V$ of $p$ contains no idempotents in $S$. Thus in this case the set $Q$ consists entirely of recurrent points.

(4) We will see that when we make the additional assumption that there exists $y_{0} \in Y$ such that $y_{0} t$ is a transitive point of $Y$ for every $t \in T$, Proposition 6.4 does allow us to conclude that the set of points in $Y$ which are $S$-recurrent is a residual subset of $Y$.

\subsection{Lemma. Assume}

(1) $(Y, T)$ is a compact metric flow,

(2) there exists $y_{0} \in Y$ such that $y_{0} r$ is a transitive point for every $r \in T$.

Then $K=\{y \in Y \mid \overline{y T}=Y\}$ is a residual subset of $Y$.

Proof. Let $W, U$ be non-empty open subsets of $Y$. There exist $r, t \in T$ with $y_{0} r \in U$ and $y_{0} r t \in W$. Hence $R_{t}^{-1}(W) \cap U \neq \emptyset$. This implies that

$$
\overline{\bigcup\left\{R_{t}^{-1}(W) \mid t \in T\right\}}=Y \text {. }
$$

Now let $\left\{W_{i} \mid i=1,2, \ldots\right\}$ be a base for the topology on $Y$,

$$
y \in P=\bigcap_{i=1}^{\infty} \bigcup\left\{R_{t}^{-1}\left(W_{i}\right) \mid t \in T\right\}
$$

and $U \subset Y$ a non-empty open subset of $Y$. Choose $W_{i} \subset U$. Then $y \in R_{t}^{-1}\left(W_{i}\right) \subset$ $R_{t}^{-1}(U)$. Thus $y t \in U$ and so the residual set $P$ is contained in $K$. 
6.7. Proposition. Assume

(1) $p^{2}=p \in \beta S \backslash S$,

(2) $V$ is a neighborhood of $p$ in $\beta S$,

(3) $Y$ is a closed, metrizable, $T$-invariant subset of $X$ such that the almost periodic points of $(Y, T)$ are dense in $Y$,

(4) there exists $y_{0} \in Y$ such that $y_{0} r$ is a transitive point for every $r \in T$.

Then $R=\left\{y \in Y \mid y q=y\right.$ for some $\left.q^{2}=q \in V \backslash S\right\}$ is a residual subset of $Y$. In particular the set of $S$-recurrent points in $Y$ is a residual subset of $Y$.

Proof. Let $K=\{y \in Y \mid \overline{y T}=Y\}$, so by the previous lemma $K$ is a residual subset of $Y$. First suppose that there exists a neighborhood $N$ of $p$ such that $y s \neq y$ for all $y \in K$ and $s \in N \cap S$. We may assume that $N$ is closed and that $N \subset V$. Applying Proposition 6.4 to $N$, we see that $Q=\left\{y \in Y \mid y q=y\right.$ for some $\left.q^{2}=q \in N\right\}$ is a residual subset of $Y$. Thus $K \cap Q$ is also a residual subset of $Y$, but $K \cap Q \subset R$ so the proof is complete in this case.

Now suppose that for any neighborhood $N$ of $p$, there exist $y_{N} \in K, s_{N} \in N \cap S$, with $y_{N} s_{N}=y_{N}$. Then $y_{N} t s_{N}=y_{N} s_{N} t=y_{N} t$ for all $t \in T$ so that $y s_{N}=y$ for all $y \in Y$. It follows that $y p=y$ for all $y \in Y$, and so in this case $R=Y$.

6.8. Remark. Notice that if $Y$ is metrizable minimal under $T$, then assumptions (3) and (4) of Proposition 6.7 are satisfied, so the set of points in $Y$ which are $S$-recurrent is a residual subset of $Y$.

We now wish to derive some multi-recurrence results from 6.7 in the case where $S$ is commutative. First we note that the first assumption of 6.7 is satisfied when $S$ is a group. In fact except in certain pathological examples, such as the 0-semigroup example of 1.10 , this assumption is also satisfied when $S$ is a commutative semigroup. This motivates the following digression.

6.9. Proposition. Let

(1) $G$ be a commutative semigroup,

(2) $I \subset \beta G$ be a minimal ideal,

(3) $I \cap G \neq \emptyset$.

Then $I \subset G$ is a finite group and $I$ is the only minimal subset of $\beta G$.

Proof. We begin by showing that $I$ is a finite subgroup of $\beta G$. Let $r \in I \cap G$ and suppose $u, v \in I$ are idempotents in $I$. Then $r=u r=r u \in I u$; similarly $r \in I v$. Hence it follows from 4.2 that $u=v$ and $I=I u$ is a group. Now

$$
I=r I=I r=\bigcup_{p \in I} L_{p}(r)
$$

Since the left multiplication map $L_{p}: I \rightarrow I$ is a homeomorphism, and $r \in G, L_{p}(r)$ is open for every $p \in I$. Thus $I$ is finite.

To see that $I \subset G$ note that since $r G \subset \overline{r G}=I$ is finite, $r G$ is finite. Thus $I=\overline{r G}=r G \subset G$.

Now suppose that $K \subset \beta G$ is any minimal ideal, and let $v \in K$ be an idempotent. Then by $4.6 v \sim u$ where $u$ is the unique idempotent in $I$. Thus $u=u v=v u=v$ (here we are using that $G$ is commutative and that $u \in G$ ) and it follows that $K=I$.

Now we proceed to derive some multi-recurrence results. 


\subsection{Multi-Recurrence Theorem I. Let}

(1) $G$ be an infinite commutative semigroup such that $\beta G \backslash G$ contains at least one idempotent,

(2) $Z$ be a compact metric space on which $G$ acts,

(3) $\lambda_{i}: G \rightarrow G, i \in \mathbb{N}$, be homomorphisms.

Let $X=Z^{\infty}, T$ be the diagonal of $G^{\infty}$ and $S=G$. For $x=\left(z_{i} \mid i \in \mathbb{N}\right) \in X$, $t=\left(g_{i} \mid i \in \mathbb{N}\right) \in T$ with $g_{i}=g$ for all $i \in \mathbb{N}$, and $s \in S$, define (right) actions of $T$ and $S$ on $X$ by

$$
\begin{aligned}
& x t=\left(z_{i} g \mid i \in \mathbb{N}\right) \text { and } \\
& x s=\left(z_{i} \lambda_{i}(s) \mid i \in \mathbb{N}\right) .
\end{aligned}
$$

Let $Y \subseteq Z^{\infty}$ be a closed, non-empty $T$-invariant subset such that there exists $y_{0} \in Y$ such that $y_{0} t$ is a transitive point of $(Y, T)$ for all $t \in T$. Assume further that the almost periodic points of $(Y, T)$ are dense in $Y$. Then the set of $S$ recurrent points of $Y$ is residual in $Y$.

Proof. With $T$ and $S$ as above, we shall now verify the hypothesis 6.1. Assumptions (1) and (2) are clear and (3) follows from the fact that $G$ is commutative. With regard to (4) let $u$ be a minimal idempotent in $\bar{T}$ and $p^{2}=p \in \overline{S T}$ with $p<u$. Then $u=\left(v_{i} \mid i \in \mathbb{N}\right)$ with $v_{i}=v$ for all $i \in \mathbb{N}$, and $p=\left(p_{i} \mid i \in \mathbb{N}\right)$ where $v$ is a minimal idempotent in $\bar{G}$ and $p_{i}^{2}=p_{i} \in \bar{G}$, for all $i \in \mathbb{N}$. Now $p<u$ implies $p_{i}<v$ whence $v<p_{i}$, for all $i \in \mathbb{N}$ and so $u<p$. Now an application of 6.7 completes the proof. (Here is where the assumption that $\beta G \backslash G$ contains an idempotent is used.)

6.11. Remark. If we assume $(Z, \mathbb{N})$ has a dense set of almost periodic points and for some $z_{0} \in Z, z_{0} n$ is a transitive point for all $n \in \mathbb{N}$, then letting $Y$ equal the diagonal, 6.10 gives us the "classical" multiple Birkhoff recurrence theorem with a hypothesis weaker than minimality of $(Z, \mathbb{N})$.

The following formulation of the multi-recurrence result (see $[\mathrm{BH}]$ ) generalizes the MBR theorem (Theorem 2.6 of $[\mathrm{F}]$ ).

\subsection{Multi-Recurrence Theorem II. Let}

(1) $S$ be an infinite commutative semigroup such that $\beta S \backslash S$ contains at least one idempotent,

(2) $Z$ be a compact metric space and $\left(Z, S, \pi_{i}\right),(1 \leq i \leq k)$ be flows on $Z$, with $\varphi_{i}: S \rightarrow Z^{Z}$ the corresponding homomorphisms (as in 1.12),

(3) $\varphi_{i}\left(s_{1}\right) \varphi_{j}\left(s_{2}\right)=\varphi_{j}\left(s_{2}\right) \varphi_{i}\left(s_{1}\right)$ for all $1 \leq i, j \leq k$ and $s_{1}, s_{2} \in S$,

(4) $G=\varphi_{1}(S) \varphi_{2}(S) \cdots \varphi_{k}(S)$,

(5) $Y \subset Z$ with $(Y, G)$ minimal.

Then the set

$\left\{x \in Y \mid\right.$ there exists $p^{2}=p \in \beta S \backslash S$ such that $x \varphi_{i}(p)=x$ for all $\left.1 \leq i \leq k\right\}$

of points of $Y$ that are simultaneously $S$-recurrent under all of the $S$-actions, is a residual subset of $Y$.

Proof. Let $X=Y^{k}, T$ be the diagonal of $G^{k}$ and define actions of $T$ and $S$ on $X$ by

$$
\begin{aligned}
& x t=\left(z_{1} g, \ldots, z_{k} g\right) \text { and } \\
& x s=\left(\pi_{1}\left(z_{1}, s\right), \ldots, \pi_{k}\left(z_{k}, s\right)\right)=\left(z_{1} \varphi_{1}(s), \ldots, z_{k} \varphi_{k}(s)\right),
\end{aligned}
$$


where $x \equiv\left(z_{1}, \ldots, z_{k}\right)$. Let $\hat{Y}=\left\{\left(z_{1}, \ldots, z_{k}\right) \mid z_{1}=z_{2}=\cdots=z_{k} \in Y\right\}$. Then $(\hat{Y}, T)$ is minimal so the assumptions of 6.7 are satisfied; note that (4) of 6.1 is satisfied because $S T=T$ in this case. Now the proof proceeds exactly as in 6.10 and the details are left to the reader.

When $G$ is an abelian group, yet another multi-recurrence result is given by the following.

\subsection{Theorem (Dynamical van der Waerden). Let}

(1) $G$ be an infinite abelian group,

(2) $(Z, G)$ be a minimal metrizable flow,

(3) $V$ be a non-empty open subset of $Z$,

(4) $g_{1}, \ldots, g_{k} \in G$.

Then there exists $n \in \mathbb{N}$ with $V \cap V g_{1}^{n} \cap \cdots \cap V g_{k}^{n} \neq \emptyset$.

Proof. In this case we set $X=Z^{k}, T=$ the diagonal of $G^{k}, S=\left\{\left(g_{1}^{-n}, \ldots, g_{k}^{-n}\right) \mid\right.$ $n \in \mathbb{N}\}$ and $Y=$ the diagonal of $Z^{k}$. If $S$ is finite, then there exists $n \in \mathbb{N}$ with $g_{1}^{n}=g_{2}^{n}=\cdots=g_{k}^{n}=e$ in which case the result clearly holds. On the other hand if $S$ is infinite, then $\beta S \backslash S$ is isomorphic to $\beta \mathbb{N} \backslash \mathbb{N}$ and hence contains an idempotent. Thus we can apply 6.7 to obtain $y=(z, \cdots, z) \in V^{k}$ which is $S$ recurrent. Hence there exists $n \in \mathbb{N}$ with $(z, \ldots, z)\left(g_{1}^{-n}, \ldots, g_{k}^{-n}\right) \in V^{k}$. Thus $z \in V$ and $z g_{i}^{-n} \in V(1 \leq i \leq k)$, whence $z \in V \cap V g_{1}^{n} \cap \cdots \cap V g_{k}^{n}$.

We now eliminate the condition that $Z$ be metrizable in 6.13 .

\subsection{Theorem. Let}

(1) $G$ be an infinite abelian group.

(2) $(Z, G)$ a minimal flow.

(3) $V$ a non-empty open subset of $Z$.

(4) $g_{1}, \ldots, g_{k} \in G$.

Then there exists $n \in \mathbb{N}$ with $V \cap V g_{1}^{n} \cap \cdots \cap V g_{k}^{n} \neq \emptyset$.

Proof. Let $W$ be a non-empty open subset of $Z$ with $\bar{W} \subset V$, and $f: Z \rightarrow$ $[0,1]$ be such that $f(x)=1$ for all $x \in W$ and $f(x)=0$ for all $x \in Z \backslash V$. Let $d(x, y)=|f(x)-f(y)|$ for all $x, y \in Z$. Then $d$ is a continuous psuedo-metric on $Z$. Hence by Proposition 1.6 of [E1], there exists a countable subgroup $K$ of $G$ with $\left\{g_{1}, \ldots, g_{k}\right\} \subset K$, such that the flow $(Z / R, K)$ is a compact metric minimal flow, where

$$
R=\{(x, y) \mid d(x k, y k)=0 \text { for all } k \in K\} .
$$

The metric $\sigma$ on $Z / R$ is given by

$$
\sigma(a, b)=\sum_{1}^{\infty} 2^{-i} d\left(x t_{i}, y t_{i}\right)
$$

where $\pi(x)=a, \pi(y)=b, K=\left\{e=t_{1}, t_{2}, \ldots\right\}$, and $\pi$ is the canonical map $Z \rightarrow Z / R$.

Let $x \in W, a=\pi(x)$, and $P=\left\{b \mid \sigma(a, b)<\frac{1}{4}\right\}$. Then $P$ is a non-empty open subset of $Z / R$, whence by 6.13 there exist $n \in \mathbb{N}$ and $b \in Z / R$ with

$$
b \in P \cap P g_{1}^{n} \cap \cdots \cap P g_{k}^{n} .
$$


Let $\pi(y)=b$. Then $\pi\left(y g_{i}^{-n}\right)=\pi(y) g_{i}^{-n}=b g_{i}^{-n} \in P$, and hence

$$
\frac{1}{4}>\sigma\left(a, b g_{i}^{-n}\right)=\sum_{1}^{\infty} 2^{-i} d\left(x t_{i}, y g_{i}^{-n} t_{i}\right)>\frac{1}{2} d\left(x, y g_{i}^{-n}\right) .
$$

Thus $\left|f(x)-f\left(y g_{i}^{-n}\right)\right|<\frac{1}{2}$, and so $y g_{i}^{-n} \in V$, i.e. $y \in V g_{i}^{n}$.

A similar argument shows that $y \in V$, completing the proof.

Now we shall indicate how to derive coloring results from our setup. First we will apply 6.14 to a minimal subset of $\beta G$ where $G$ is an infinite abelian group, to obtain a version of the classical van der Waerden theorem on finite colorings (see BPT] $)$. Then we will apply 6.3 to obtain an IP generalization to the case where $\beta G \backslash G$ contains an invariant set. We refer to this generalization as the combinatorial van der Waerden theorem.

\subsection{Classical van der Waerden Theorem. Let}

(1) $(G,+)$ be an infinite abelian group (for example $G=\mathbb{Z}$ or $\mathbb{Z}^{r}$ ),

(2) $U_{1}, \ldots, U_{l}$ be a finite partition of $G$.

Then there exists some $m$, with $1 \leq m \leq l$ such that given any finite subset $F$ of $G$, there exists $n \in \mathbb{N}$ and $b \in U_{m}$ such that

$$
b+n a \in U_{m}, \quad \text { for all } a \in F,
$$

(where $n a=a+\cdots+a, n$ times).

Proof. Let $M$ be a minimal subset of $\beta G$. Then $M \subset \bar{U}_{1} \cup \cdots \cup \bar{U}_{l}=\beta G$ so there exists $m$ with $V \equiv \bar{U}_{m} \cap M \neq \emptyset$. Now for any finite subset $F=\left\{a_{1}, \ldots, a_{k}\right\}$ we apply 6.14 to the minimal flow $(M, G)$, open set $V$, and elements $\left\{-a_{1}, \ldots,-a_{k}\right\} \subset$ $G$ obtaining an element

$$
b \in V \cap V-n a_{1} \cap \cdots \cap V-n a_{k} \subset \bar{U}_{m} \cap \bar{U}_{m}-n a_{1} \cap \cdots \cap \bar{U}_{m}-n a_{k} .
$$

Since $\bar{U}_{m}$ is both open and closed in $\beta G$, it follows that $U_{m} \cap U_{m}-n a_{1} \cap \cdots \cap U_{m}-$ $n a_{k} \neq \emptyset$, which completes the proof.

\subsection{Combinatorial van der Waerden Theorem. Let}

(1) $(G,+)$ be an infinite commutative semigroup,

(2) $\beta G \backslash G$ contain an invariant set (for example $G=\mathbb{N}$ or $\mathbb{N}^{r}$ ),

(3) $U_{1}, \ldots, U_{p}$ be a finite partition of $G$.

Then there exists some $m$, with $1 \leq m \leq p$ such that given any finite subset $F$ of $G$ and any injective map $L: \mathbb{N} \rightarrow \mathbb{N}$, there exists $n \in \pi(L)$ and $b \in U_{m}$ such that

$$
b+n a \in U_{m}, \quad \text { for all } a \in F,
$$

(where $n a=a+\cdots+a, n$ times).

We remark that in this theorem $G$ is not assumed to be countable and the integer $m$ is independent of the IP-set $\pi(L)$.

Proof. Let $u^{2}=u \in \beta G \backslash G$ be a minimal idempotent (here we are using assumption (2)). Now $\beta G=\overline{U_{1}} \cup \cdots \cup \bar{U}_{p}$ so $u \in \overline{U_{m}}$ for some $m$. Write $V=\bar{U}_{m}$ and note that $V$ is a neighborhood of $u$. 
Now let $F=\left\{a_{1}, \ldots, a_{k}\right\} \subset G$ be any finite set. Set $X=(\beta G)^{k}, T=$ $\{(g, \ldots, g) \mid g \in G\}$ and let $S=\mathbb{N}$. Define right $S$ and $T$ actions on $X$ by setting

$$
\begin{aligned}
x n & \equiv\left(z_{1}, \ldots, z_{k}\right) n=\left(z_{1}+a_{1} n, \cdots, z_{k}+a_{k} n\right), \\
x t & \equiv\left(z_{1}, \ldots, z_{k}\right)(g, \ldots, g)=\left(z_{1}+g, \cdots, z_{k}+g\right) .
\end{aligned}
$$

Consider the idempotent $\bar{u}=(u, \ldots, u)$. By exactly the same reasoning used in 6.10 one verifies the hypothesis 6.1 . Now apply 6.3 with $\mathcal{H}=\left\{\pi\left(L^{n}\right) \mid n \in \mathbb{N}\right\}$ and the neighborhood $V^{k} \equiv V \times \cdots \times V$ of $\bar{u}$. Hence there exist $n \in \pi(L)$ and $b \in V$ such that $\left(b+a_{1} n, \ldots, b+a_{k} n\right) \in V^{k}$.

\section{Appendix A}

In this appendix we give an explicit construction of a Stone-Čech compactification of the semigroup $T$ when it is given the discrete topology. The underlying space, $\beta T$ of this construction will be the collection of all ultra-filters on $T$. The set $\beta T$ will be provided with a compact Hausdorff topology such that the properties stated in Definition 2.1 are satisfied when $T$ is identified with the set of principal ultra-filters on $T$. In addition a semigroup structure on $\beta T$ will be defined and it will be shown that it has the properties deduced abstractly in section 2 . We hope thereby to provide more insight into what is going on. (A good reference for the material on filters and ultra-filters is Bourbaki: Eléments de Mathématique, Topologie Générale, Livre III, Chapitre I [B].)

A.1. Definition. Let $X$ be a set and $\mathcal{F} \subset \mathcal{P} X$, the collection of subsets of $X$. Then:

(1) $\mathcal{F}$ is a filter on $X$ if
(a) $\emptyset \notin \mathcal{F}$,
(b) $A, B \in \mathcal{F}$ implies $A \cap B \in \mathcal{F}$,
(c) $A \in \mathcal{F}$ and $A \subset B$ implies $B \in \mathcal{F}$;

(2) $\mathcal{F}$ is a filter base on $X$ if
(a) $\emptyset \notin \mathcal{F}$,
(b) given $A, B \in \mathcal{F}$ there exists $C \in \mathcal{F}$ with $C \subset A \cap B$;

(3) $\mathcal{F}$ is an ultra-filter on $X$ if $\mathcal{F}$ is a maximal filter on $X$; i.e if $\mathcal{G}$ is a filter on $X$ with $\mathcal{F} \subset \mathcal{G}$, then $\mathcal{F}=\mathcal{G}$.

A.2. Remarks. (1) Let $\mathcal{F} \subset \mathcal{P} X$ have the finite intersection property (F.I.P.), i.e. any finite collection of elements of $\mathcal{F}$ has a non-empty intersection. Then the collection of all these finite intersections forms a filter base on $X$.

(2) Let $\mathcal{F}$ be a filter base on $X$. Then $\mathcal{H}=\{H \mid F \subset H$ for some $F \in \mathcal{F}\}$ is a filter on $X$. It is the "smallest" filter on $X$ containing $\mathcal{F}$, and is called the filter generated by $\mathcal{F}$ (Notation: $\mathcal{H} \equiv \mathcal{F} \subset$ )

(3) A filter base is called an ultra-filter base if the filter it generates is an ultrafilter.

A characterization of ultra-filters is given by (see $[\bar{B}]$ ):

A.3. Proposition. Let $\mathcal{F}$ be a filter on $X$. Then the following are pairwise equivalent:

(1) $\mathcal{F}$ is an ultrafilter,

(2) $A \in \mathcal{F}$ or $A^{\prime} \in \mathcal{F}$ for all $A \subset X$,

(3) if $A_{1} \cup \cdots \cup A_{n} \in \mathcal{F}$, then $A_{i} \in \mathcal{F}$ for some $i$. 
A.4. Definition. (1) The set of all ultra-filters on $T$ is denoted by $\beta T$.

(2) For $\emptyset \neq A \subset T, h(A)$ will denote the set of ultra-filters containing $A$. We write $h(t)$ instead of $h(\{t\})$ for $t \in T$.

(3) Let $\Gamma \subset \beta T$. Then $\Gamma \in \mathcal{T}$ if given $p \in \Gamma$ there exists $A \in p$ with $h(A) \subset \Gamma$.

A.5. Proposition. The collection $\mathcal{T}$ defines a compact Hausdorff topology on $\beta T$.

Proof. We first show that $\mathcal{T}$ is a topology on $\beta T$. Clearly $\emptyset$ and $\beta T$ are in $\mathcal{T}$. Let $\Gamma_{1}, \Gamma_{2} \in \mathcal{T}$ and $p \in \Gamma_{1} \cap \Gamma_{2}$. Then there exist $A_{i} \in p$ with $h\left(A_{i}\right) \subset \Gamma_{i}(i=1,2)$. Then $A_{1} \cap A_{2} \in p$ and $h\left(A_{1} \cap A_{2}\right) \subset \Gamma_{1} \cap \Gamma_{2}$. Hence $\mathcal{T}$ is closed under finite intersections. Now let $\Gamma_{i} \in \mathcal{T}(i \in I)$ and $p \in \Gamma=\bigcup \Gamma_{i}$. Then $p \in \Gamma_{i}$ for some $i$, and so $h(A) \subset \Gamma_{i} \subset \Gamma$ for some $A \in p$.

To see that $\mathcal{T}$ is Hausdorff, let $p, q \in \beta T$ with $p \neq q$. There exists $A \subset T$ with $A \in p$ and $A^{\prime} \in q$. Then $h(A)$ and $h\left(A^{\prime}\right)$ are in $\mathcal{T}$ with $p \in h(A), q \in h\left(A^{\prime}\right)$ and $h(A) \cap h\left(A^{\prime}\right)=\emptyset$.

Finally we show that $\mathcal{T}$ is compact. Let $\left(\Gamma_{i} \mid i \in I\right)$ be a family of open sets whose union is $\beta T$. Without loss of generality we may assume that $\Gamma_{i}=h\left(A_{i}\right)$ with $\emptyset \neq A_{i} \subset T$ for all $i \in I$. Let $\mathcal{I}$ be the collection of finite subsets of $I$ and assume $A_{F} \neq \beta T$ where $A_{F}=\bigcup\left\{h\left(A_{i}\right) \mid i \in F\right\}$ for all $F \in \mathcal{I}$. Then the family $\left(A_{i}{ }^{\prime} \mid i \in I\right)$ has the finite intersection property and so there exists $p \in \beta T$ with $A_{i}{ }^{\prime} \in p$ for all $i \in I$. But this contradicts the fact that $\beta T=\bigcup\left\{h\left(A_{i}\right) \mid i \in I\right\}$.

A.6. Proposition. Let $\phi: T \rightarrow \beta T$ be such that $\phi(t)=h(\{t\}) \equiv$ the principal ultra-filter generated by $t(t \in T)$. Then $\overline{\phi(A)}=h(A)(A \subset T)$.

Proof. If $A=\emptyset$, then $\overline{\phi(A)}=\bar{\emptyset}=\emptyset=h(A)$.

Let $A \neq \emptyset, p \in \overline{\phi(A)}$ and $U \in p$. Then there exists $t \in A$ with $\phi(t) \in h(U)$. Thus $A \cap U \neq \emptyset(U \in p)$ and so $A \in p$ and $p \in h(A)$.

Now let $p \in h(A)$ and $\Gamma$ be a neighborhood of $p$. Then there exists $U \in p$ with $h(U) \subset \Gamma$. Then $U \cap A \neq \emptyset$ and $U \in \phi(t)(t \in A \cap U)$ and so $\phi(A) \cap \Gamma \neq \emptyset$.

A.7. Remarks. (1) The map $\phi$ defined above is one-one;

(2) $\overline{\phi(T)}=h(T)=\beta T$;

(3) $h\left(A^{\prime}\right)=(h(A))^{\prime}$;

(4) $\beta T=h(T)=h\left(A \cup A^{\prime}\right)=h(A) \cup h\left(A^{\prime}\right)=h(A) \cup(h(A))^{\prime}$. Thus every set of the form $h(A)$ is both open and closed;

(5) $\phi(t)$ is both open and closed for all $t \in T$;

(6) $\phi(T)$ is an open dense subset of $\beta T$.

A.8. Proposition. Let $\Gamma$ be an open subset of $\beta T$ and $A=\{t \mid \phi(t) \in \Gamma\}$. Then $\bar{\Gamma}=h(A)$. Thus the closure of every open set is both open and closed. (Spaces having this property are called extremely disconnected.)

Proof. Since $\Gamma$ is open and $\overline{\phi(T)}=\beta T, \bar{\Gamma}=\overline{\phi(T) \cap \Gamma}=\overline{\phi(A)}=h(A)$.

A.9. Lemma. Let

(1) $f: T \rightarrow X$,

(2) $p \in \beta T$,

(3) $f(p)=\{f(A) \mid A \in p\}$.

Then $f(p)$ is an ultrafilter base on $X$. 
Proof. Let $A, B \in p$. Then $A \cap B \in p, f(A \cap B) \subset f(A) \cap f(B)$ and $f(A \cap B) \in f(p)$ shows that $f(p)$ is a filter base on $X$.

Now let $Y \subset X$. Then $T=f^{-1}(X)=f^{-1}(Y) \cup f^{-1}\left(Y^{\prime}\right) \in p$ implies that $f^{-1}(Y)$ or $f^{-1}\left(Y^{\prime}\right)=\left(f^{-1}(Y)\right)^{\prime} \in p$. Since $f\left(f^{-1}(Y)\right) \subset Y$ and $f\left(f^{-1}\left(Y^{\prime}\right)\right) \subset Y^{\prime}, Y$ or $Y^{\prime}$ is in $f(p)^{\subset}$.

A.10. Definition. Let $\mathcal{F}$ be a filter base on the topological space, $X$. Then $\mathcal{F}$ converges to $x \in X(\mathcal{F} \rightarrow x)$ if $\mathcal{F} \subset$ contains the neighborhood filter $\mathcal{N}_{x}$ of $x$.

The following characterization of compact Hausdorff spaces is standard.

A.11. Proposition. The space $X$ is compact Hausdorff if and only if every ultrafilter base on $X$ converges to a unique point of $X$.

A.12. Proposition. Let $X$ be compact Hausdorff and $f: T \rightarrow X$. Then there exists a continuous funtion $\hat{f}: \beta T \rightarrow X$ such that $f(t)=\hat{f}(\phi(t))(t \in T)$. (Thus the pair $(\beta T, \phi)$ is a Stone-Cech compactification of T.)

Proof. By A.9 and A.11 for every $p \in \beta T$ there is a unique point $\hat{f}(p)=x$ in $X$ such that $f(p) \rightarrow x$. Clearly $f(\phi(t)) \rightarrow f(t)$ whence $\hat{f}(\phi(t))=f(t)(t \in T)$.

Let $\hat{f}(p)=x$ and let $U$ be a closed neighborhood of $x$. Then there exists $A \in p$ with $f(A) \subset U$.

Now let $q \in h(A), y=\hat{f}(q)$, and $V$ a neighborhood of $y$. Since $f(q)$ converges to $y, f^{-1}(V) \in q$ and so there exists $a \in A \cap f^{-1}(V)$. Then $f(a) \in U \cap V$ whence $y \in \bar{U}=U$. Consequently $\hat{f}(h(A)) \subset U$, and so $\hat{f}$ is continuous.

A.13. Remarks. (1) Since $\phi$ is one-one, we usually identify $\phi(t)$ with $t(t \in T)$.

(2) The properties stated in 2.2 follow immediately from A.7 and A.8.

A.14. Definition. We should now like to define the product $p q$ of two elements $p, q$ of $\beta T$ in terms of the ultrafilters $p$ and $q$. To this end for $A \subset T$ and $p \in \beta T$ set $A p=\{t \in T \mid$ there exists $B \in p$ with $B t \subset A\}$.

A.15. Lemma. Let $A \subset T$ and $t \in T$. Then $A h(t)=\{r \mid \operatorname{tr} \in A\}$. Thus if $T$ is a group, $A h(t)=t^{-1} A$.

Proof. Note that $r \in A h(t)$ if and only if there exists $B \in h(t)$ with $B r \subset A$ if and only if $t r \in A$.

A.16. Proposition. Let $p \in \beta T, A, B \subset T$. Then

(1) $\emptyset p=\emptyset, T p=T$,

(2) $A \subset B$ implies $A p \subset B p$,

(3) $(A \cap B) p=A p \cap B p$,

(4) $(A \cup B) p=A p \cup B p$,

(5) $(A p)^{\prime}=A^{\prime} p$.

Proof. (1) and (2) are clear.

(3) By (2) $(A \cap B) p \subset A p \cap B p$. Now let $t \in A p \cap B p$. There exist $C_{1}, C_{2} \in p$ with $C_{1} t \subset A, C_{2} t \subset B$. Then $C_{1} \cap C_{2} \in p$ and $\left(C_{1} \cap C_{2}\right) t \subset A \cap B$, whence $t \in(A \cap B) p$.

(4) Again by (2), $A p \cup B p \subset(A \cup B) p$. Let $t \in(A \cup B) p$. There exists $C \in p$ with $C t \subset A \cup B$. Set $C_{1}=\{s \in C \mid$ st $\in A\}$ and $C_{2}=\{s \in C \mid s t \in B\}$. Then $C=C_{1} \cup C_{2}$ whence one of them, say $C_{1}$ is in $p$. Hence $C_{1} t \subset A$ whence $t \in A p \subset A p \cup B p$. 
(5) By (4) and (1) $A p \cup A^{\prime} p=\left(A \cup A^{\prime}\right) p=T p=T$. By 3 and $1 A p \cap A^{\prime} p=$ $\left(A \cap A^{\prime}\right) p=\emptyset p=\emptyset$. Thus $A^{\prime} p=(A p)^{\prime}$.

A.17. Lemma. Let $p, q \in \beta T$ and set $r=\{A \subset T \mid A p \in q\}$. Then $r \in \beta T$.

Proof. This follows immediately from A.16.

Lemma A.17 now allows us to define the product of two ultra-filters. Thus:

A.18. Definition. Let $p, q \in \beta T$. Then $p q=\{A \subset T \mid A p \in q\}$.

A.19. Lemma. Let $\emptyset \neq A \subset T$, and $p \in \beta T$. Then

(1) $t \in A p$ iff $A \in p h(t)$,

(2) $h(t s)=h(t) h(s)$.

Proof. (1) Notice that $t \in A p$ iff $A p \in h(t)$ iff $A \in p h(t)$.

(2) Let $\emptyset \neq A \subset T$. Then by A.15, $A h(t)=\{r \mid t r \in A\}$ and so $s \in A h(t)$ iff $t s \in A$ iff $A \in h(t s)$. But $s \in A h(t)$ iff $A h(t) \in h(s)$ iff $A \in h(t) h(s)$.

A.20. Proposition. Let $t \in T, p \in \beta T$. Then the maps $L_{p}, R_{t}: \beta T \rightarrow \beta T$ are continuous, where $L_{p}(q)=p q$ and $R_{t}(q)=q h(t)(q \in \beta T)$.

Proof. Since the set $\{h(s) \mid s \in T\}$ is dense in $\beta T$ (A.7), it suffices to show that if $\left(h\left(s_{\alpha}\right)\right)$ is a net converging to $q$, then $p h\left(s_{\alpha}\right)$ converges to $p q$ and $h\left(s_{\alpha}\right) h(t)$ converges to $q h(t)$.

Now let $N$ be a neighborhood of $p q$. Then there exists $A \subset T$ with $p q \in h(A) \subset$ $N$. Then $A \in p q$ whence $A p \in q$ and so $h(A p)$ is a neighborhood of $q$. Therefore there exists $\beta$ with $h\left(s_{\alpha}\right) \in h(A p)(\alpha>\beta)$. Thus $A p \in h\left(s_{\alpha}\right)$ whence by (1) of A.19 $A \in p h\left(s_{\alpha}\right)$ so that $p h\left(s_{\alpha}\right) \in h(A) \subset N(\alpha>\beta)$.

To see that $R_{t}$ is continuous let $L$ be a neighborhood of $q h(t)$. Then there a subset $B$ of $T$ with $q h(t) \in h(B) \subset L$. Then $B \in q h(t)$ and so by A.19 $t \in B q$. Consequently, $C t \subset B$ for some $C \in q$. This implies that $h(C)$ is a neighborhood of $q$ whence there exists $\beta$ with $h\left(s_{\alpha}\right) \in h(C)(\alpha>\beta)$. Thus $s_{\alpha} \in C$ and $s_{\alpha} t \in$ $B(\alpha>\beta)$. Finally $h\left(s_{\alpha}\right) h(t)=h\left(s_{\alpha} t\right) \in h(B) \subset L(\alpha>\beta)$.

A.21. Proposition. Let $p, q, r \in \beta T$, and $A \subset T$. Then

(1) $(p q) r=p(q r)$,

(2) $(A p) q=A(p q)$.

Proof. (1) Let $p=h\left(t_{1}\right), q=h\left(t_{2}\right)$, and $r=h\left(t_{3}\right)$. Then it follows immediately from A.19 that $(p q) r=p(q r)$ in this case. The general case now follows from A.20.

(2) Notice that $: t \in(A p) q$ iff $(A p) q \in h(t)$ iff (by A.18) $A p \in q h(t)$ iff (by A.18) $A \in p(q h(t))$ iff (by 1 above) $A \in(p q) h(t)$ iff (by A.18) $A(p q) \in h(t)$ iff $t \in A(p q)$.

A.22. Remarks. (1) Although it is natural to identify $\phi(t)=h(t)$ with $t$, one should be careful not to equate $A t=\{s t \mid s \in T\}$ with $A h(t)=\{s \mid t s \in A\}$ (= $t^{-1} A$ when $T$ is a group) nor to equate $\phi(A)=\{\phi(t) \mid t \in A\}$ with $h(A)=\overline{\phi(A)}$ for arbitrary subsets $A$ of $T$.

(2) However, let $p \in \beta T, t \in T$ and set $p t=\{A t \mid A \in p\}$. Then $p t$ is the image of the ultrafilter $p$ under the map $R_{t}: T \rightarrow T$, whence by A.9 $p t$ is an ultrafilter base on $T$. Now if we identify $s$ with $\phi(s)(s \in T)$, pt becomes an ultrafilter base on $\beta T$ and so by A.11 it converges to some point $q \in \beta T$. Claim $q=p h(t)$. To see this, let $\Gamma$ be a closed neighborhood of $q$. Then there 
exists $A \in p$ with $A t=\phi(A t) \subset \Gamma$ and so $h(A t)=\overline{\phi(A t)} \subset \Gamma$. Since $A \in p$, $p t \in \Gamma$.

(3) Proposition A.16 shows that the maps $A \rightarrow A p: \mathcal{P}(T) \rightarrow \mathcal{P}(T)$ are Boolean algebra homomorphisms $(p \in \beta T)$, and Proposition A.17 states that the product in $\beta T$ corresponds to composition of maps.

(4) Let $T$ be a group, $A \subset T, t \in T$, and $p \in \beta T$. Then $(A t) p=(A p) t$.

To see this note that $s \in(A t) p$ iff $B s \subset A t$ for some $B \in p$ iff $B \subset A t s^{-1}$ for some $B \in p$ iff $A t s^{-1} \in p$.

On the other hand $s \in(A p) t$ iff $s t^{-1} \in A p$ iff $B s t^{-1} \subset A$ for some $B \in p$ iff $B \subset A t s^{-1}$ for some $B \in p$ iff $A t s^{-1} \in p$.

(5) The preceding remark does not hold for arbitrary semigroups. The 0-semigroup of Section 1 provides a counter-example. In that case it is easy to check that $A p=\emptyset$ and $\{0\} p=T$ if $0 \notin A \subset T$ and $p \in \beta T$. Then if $A$ is a non-empty subset of $T$ with $0 \notin A,(A t) p=\{0\} p=T$, but $(A p) t=\emptyset t=\emptyset$ for all $t \in T$ and $p \in \beta T$.

(6) Let $A \subset T, p, q \in \beta T$. Then $A p \subset T$ and so by A.6, $\bar{A}=h(A)$ and $\overline{A p}=$ $h(A p)$. Thus $q \in \overline{A p}$ iff $q \in h(A p)$ iff $A p \in q$ iff (by A.18) $A \in p q$ iff $p q \in \bar{A}$ iff $q \in L_{p}^{-1}(\bar{A})$. Hence in this case $\overline{A p}=L_{p}^{-1}(\bar{A})=A * p$ (see 5.8).

\section{APPENDix B}

In this appendix we shall extend and also obtain the IP version of Furstenberg's theorem on locating recurrent points (see Proposition 2.4 of $[\mathrm{F}]$ ). To compare it with the approach taken in Section 6, here we replace (4) of 6.1 by an assumption on the subset $Y$ of the flow $(X, S)$. Furstenberg's result basically says that if $Y$ is "homogenous" and has a certain "weak recurrence property" (see Proposition 2.4 in $[\mathrm{F}]$ for details) then it contains $S$ recurrent points. The following Corollary B.6 generalizes this result. To describe our assumption we begin with the following.

B.1. Definition. Let $(X, S)$ be a flow and $2^{X}$ be the collection of non-empty closed subsets of $X$. Then $S$ acts on $2^{X}$ via the map $(A, s) \rightarrow A \circ s=\{a s \mid a \in A\}$. Then as usual this action may be extended to an action of $\beta S$ on $2^{X}$. The image of $A \in 2^{X}$ under the element $p$ of $\beta S$ will be denoted by $A \circ p$.

The recurrence condition we impose on the set $A \in 2^{X}$ is : $A \subseteq A \circ p$ for some idempotent $p=p^{2} \in \beta S \backslash S$. This condition is weaker than demanding that $A \in 2^{X}$ be a recurrent point of the flow $\left(2^{X}, S\right)$. This follows at once because by Definition $5.19 A$ being a recurrent point in $2^{X}$ amounts to saying that $A=A \circ p$ for some $p \in$ $\beta S \backslash S$. We shall see that Furstenberg's hypothesis implies our recurrence condition (see Remark B.7). In fact we shall obtain an IP version of the above-mentioned result of Furstenberg (Proposition B.4). We begin by recalling the assumptions which will remain in force throughout this appendix.

B.2. Standing Assumptions. (1) Let $S$ and $T$ be infinite semigroups.

(2) Let $X$ be a compact Hausdorff space on which both $S$ and $T$ act continuously (on the right). That is $(X, T)$ and $(X, S)$ are both dynamical systems. We shall view $S$ and $T$ as subsets of $X^{X}$.

(3) Viewed as elements of $X^{X}$, st $=t s$, for all $s \in S, t \in T$.

Notice that B.2 is the same as 6.1 except that (4) has been omitted.

We begin with the following lemma. 
B.3. Lemma. Let

(1) $X$ be metrizable with metric d,

(2) $J: \mathbb{N} \rightarrow S$ be one-one,

(3) $A \in 2^{X}$ with $A \subset \overline{A J^{n}(\mathbb{N})} \subset X$ for all $n \in \mathbb{N}$ (this holds in particular if $A$ is recurrent under the sequence $\left.\left\{J_{1}, J_{2}, \ldots\right\}\right)$,

(4) $\epsilon>0$.

Then for every $n \in \mathbb{N}$ there exists an $x \in A$ and $s \in \pi\left(J^{n}\right)$ such that $d(x, x s)<\epsilon$.

Proof. Let $n \in \mathbb{N}$. First we construct a sequence $\left(a_{k}\right) \subset A$ and a subsequence $\left(s_{k}\right)$ of $\left(J_{i}^{n}\right)=\left(J_{n+i}\right)$ such that

$$
d\left(a_{k} r(k, l), a_{l}\right)<\frac{\epsilon}{2},
$$

where $r(k, l)=s_{k-1} \cdots s_{l} \in \pi\left(J^{n}\right)$ for $1 \leq l<k$.

We proceed by induction. Choose $a_{1} \in A$. Now $A \subset \overline{\left(A J^{n}(\mathbb{N})\right)}$, so there exists $a_{2} \in A$ and $s_{1} \in J^{n}(\mathbb{N})$ with $d\left(a_{2} s_{1}, a_{1}\right)<\frac{\epsilon}{2}$. Then $r(2,1)=s_{1}$ and thus $a_{1}, a_{2}$ and $s_{1}$ satisfy the required conditions.

Now assume that $a_{1}, \ldots, a_{m} \in A$, and $\left\{s_{1}, \ldots, s_{m-1}\right\}=\left\{J_{l_{1}}, J_{l_{2}}, \ldots, J_{l_{m-1}}\right\}$ with $n \leq l_{1}<l_{2}<\cdots<l_{m-1}$ and $m>1$ have been defined so that $d\left(a_{k} r(k, l), a_{l}\right)<$ $\frac{\epsilon}{2}$, for all $1 \leq l<k \leq m$. Set $\rho=\max \left\{d\left(a_{k} r(k, l), a_{l}\right) \mid 1 \leq l<k \leq m\right\}$. Let $\delta>0$ be such that

$$
\text { if } d\left(a, a_{m}\right)<\delta, \text { then } d\left(\operatorname{ar}(m, l), a_{m} r(m, l)\right)<\frac{\epsilon}{2}-\rho \text { for all } 1 \leq l<m .
$$

Choose $l>l_{m-1}$. Since $A \subset \overline{\left(A J^{l}(\mathbb{N})\right)}$ there exist $a_{m+1} \in A$ and $s_{m}=J_{l_{m}}$ with $l_{m}>l$ and $d\left(a_{m+1} s_{m}, a_{m}\right)<\min \left(\frac{\epsilon}{2}, \delta\right)$.

Now

$$
\begin{aligned}
d\left(a_{m+1} r(m+1, l), a_{l}\right) & \leq d\left(a_{m+1} r(m+1, l), a_{m} r(m, l)\right)+d\left(a_{m} r(m, l), a_{l}\right) \\
& <\frac{\epsilon}{2}-\rho+\rho=\frac{\epsilon}{2},
\end{aligned}
$$

for all $1 \leq l<m$. Hence $d\left(a_{k} r(k, l), a_{l}\right)<\frac{\epsilon}{2}$, for all $1 \leq l<k \leq m+1$.

Having constructed the sequence above, the proof is completed by observing that the compactness of $X$ implies that there exist $k, l \in \mathbb{N}$ with $k>l$ and $d\left(a_{k}, a_{l}\right)<\frac{\epsilon}{2}$. Then $d\left(a_{k} r(k, l), a_{k}\right) \leq d\left(a_{k} r(k, l), a_{l}\right)+d\left(a_{l}, a_{k}\right)<\epsilon$.

\section{B.4. Proposition. Let}

(1) $X$ be metrizable,

(2) A be a closed, non-empty $T$-invariant subset of $X$ such that the flow $(A, T)$ is minimal,

(3) $J: \mathbb{N} \rightarrow S$ be one-one,

(4) $A \in 2^{X}$ with $A \subset \overline{A J^{n}(\mathbb{N})} \subset X$ for all $n \in \mathbb{N}$ (this holds in particular if $A$ is recurrent under the sequence $\left.\left\{J_{1}, J_{2}, \ldots\right\}\right)$.

Then $Q=\left\{a \in A \mid a q=a\right.$ for some $\left.q^{2}=q \in \pi_{\infty}(J)\right\}$ is a residual subset of $A$, (compare with 6.4).

Proof. For every $n \in \mathbb{N}$ set

$$
Z_{n}=\left\{a \in A \mid d(a, a s)<\frac{1}{n}, \text { for some } s \in \pi\left(J^{n}\right)\right\} .
$$


Then $Z_{n}$ is a non-empty open subset of $A$ by B.3. Assume that $\emptyset \neq U \subset A$ is any open subset of $A$. Since $(A, T)$ is minimal, there exists a finite subset $F$ of $T$ such that

$$
\bigcup\left\{R_{t}^{-1}(U) \mid t \in F\right\}=A .
$$

Since $F$ is finite we can choose $\delta>0$ such that $d(a, b)<\delta$ implies that $d(a t, b t)<\frac{1}{n}$ for all $t \in F$. By B.3 we can find $a \in A, s \in \pi\left(J^{n}\right)$ with $d(a, a s)<\delta$. Now there exists $t \in F$ with at $\in U$. But $d($ at, ats $)=d($ at, ast $)<\frac{1}{n}$, so at $\in U \cap Z_{n}$. This implies that $Z_{n}$ is dense in $A$. Thus $Z=\bigcap Z_{n}$ is a residual subset of $A$.

To see that $Z \subset Q$ we proceed as in the last paragraph of the proof of 6.4. Let $z \in Z$. Since $z \in Z_{n}$, there exists $s_{n} \in \pi\left(J^{n}\right)$ with $d\left(z, z s_{n}\right)<1 / n$. Let $r$ be a limit point of $\left\{s_{n}\right\}$ in $\beta S$. Then $z r=z$ and $r \in \pi_{\infty}(J)$. Since $\pi_{\infty}(J)$ is a closed non-empty semigroup, there exists an idempotent $q \in \pi_{\infty}(J)$ with $z q=z$. Thus $z \in Q$.

B.5. Remark. The set $Q$ of Proposition B.4 may not contain any $S$-recurrent points. Indeed $\pi_{\infty}(J)$ may intersect $S$ or even be entirely contained in $S$. Of course if $\pi_{\infty}(J) \cap S=\emptyset$, then $Q$ consists entirely of recurrent points.

\section{B.6. Corollary. Let}

(1) $X$ be metrizable,

(2) $A$ be a closed, non-empty $T$-invariant subset of $X$ such that the flow $(A, T)$ is minimal,

(3) $p^{2}=p \in \beta S \backslash S$ with $A \subset A \circ p$,

(4) $V$ be a neighborhood of $p$.

Then $R=\left\{a \in A \mid a q=a\right.$ for some $\left.q^{2}=q \in V \backslash S\right\}$ is a residual subset of $A$, (compare with 6.4).

Proof. First suppose that for any neighborhood $N$ of $p$, there exist $a_{N} \in A, s_{N} \in$ $N \cap S$, with $a_{N} s_{N}=a_{N}$. Then $a_{N} t s_{N}=a_{N} s_{N} t=a_{N} t$ for all $t \in T$ so that $a s_{N}=a$ for all $a \in A$. It follows that $a p=a$ for all $a \in A$, and so in this case $R=A$.

Now suppose that there exists a neighborhood $N$ of $p$ such that as $\neq a$ for all $a \in A$ and $s \in N \cap S$. We may assume that $N$ is closed and that $N \subset V$. For each $n \in \mathbb{N}$ we choose a neighborhood $V_{n}$ of $p$ with $V_{n} \subset N \cap V$ such that $d_{H}(A \circ p, A s)<\frac{1}{n}$ for all $s \in V_{n} \cap S$. Here $d_{H}$ is the Hausdorff metric on $2^{X}$ induced by the metric $d$ on $X$. By 3.3 we can find $J: \mathbb{N} \rightarrow S$ with $\pi\left(J^{n}\right) \subset V_{n}$ for all $n \in \mathbb{N}$. Then for any $n \in \mathbb{N}, J_{n} \in V_{n}$ so $d\left(a, A J_{n}\right)<\frac{1}{n}$ for all $a \in A \subset A \circ p$. It follows that $A \subset \overline{A J^{n}(\mathbb{N})}$. Thus we can apply B.4 to conclude that the set

$$
R_{0}=\left\{a \in A \mid a q=a \text { for some } q^{2}=q \in \pi_{\infty}(J)\right\}
$$

is a residual subset of $A$. But $\pi_{\infty}(J)$ is contained in the closed neighborhood $V \cap N$ of $p$, so $R_{0} \subset R$ and the proof is complete.

B.7. Remark. As mentioned above, Furstenberg's hypothesis (in Proposition 2.4, F] implies assumption (3) of Corollary B.6. To see this, first we remark that in this case $S=\mathbb{N}$. Next, writing the map $S$ on the right, recall Furstenberg's hypothesis: for every $\varepsilon>0$, there are $x, y \in A$ and $n \geq 1$ such that $d\left(y S^{n}, x\right)<\varepsilon$. From this it follows easily that $z \in A \circ p$ for some $z \in A$ and $p \in \beta S \backslash S$. Since the action of the group $T$ commutes with $S$ and $A$ is $T$-invariant, we have

$$
z T \subset A T \circ p=A \circ p
$$


and since $T$ acts minimally on $A$, it follows that $A \subset A \circ p$. Moreover, since the set $\{p \mid A \subseteq A \circ p\}$ is a closed subsemigroup, without loss of generality such a $p$ can be taken to be an idempotent.

\section{REFERENCES}

[AF] J. Auslander and H. Furstenberg: Product recurrence and distal points, Trans. A.M.S. Vol. 343, No. 1 (1994), 221-232. MR 94g:54027

[BH] V. Bergelson and N. Hindman: Some topological semicommutative van der Waerden type theorems and their combinatorial consequences, J. of London Math. Soc. (2) 45 (1992), 385-403. MR 93j:05162

[BPT] A. Blaszczyk, S. Plewik and S. Turek: Topological multidimensional van der Waerden theorem, Comment. Math. Univ. Carolinae 30, 4 (1989), 783-787. MR 92a:54035

[B] N. Bourbaki: Elements de Mathematique, Topologie Generale, (1940). MR 3:55e

[E1] R. Ellis: The Furstenberg structure theorem, Pacific Journal of Mathematics, Vol. 76, No. 2, (1978), 345-349. MR 58:7574

[E2] R. Ellis: Lectures in Topological Dynamics, Benjamin, New York, (1969). MR 42:2463

[F] H. Furstenberg: Recurrence in Ergodic Theory and Combinatorial Number Theory, Princeton University Press, Princeton N.J. (1981). MR 82j:28010

[F1] H. Furstenberg: Poincaŕe recurrence and number theory, Bulletin of AMS, Vol. 5, No. 5 (1981), 211-235. MR 83d:10067

[FK] H. Furstenberg and Y.Katznelson: Idempotents in compact semigroups and Ramsey theory, Israel J. of Math. Vol. 68, No. 3 (1989), 257-270. MR 92d:05170

[FW] H. Furstenberg and B. Weiss: Topological dynamics and combinatorial number theory, Journal d'Analyse Math. 34 (1978), 61-85. MR 80g:05009

[G] S. Glasner: Topological ergodic decompositions and applications to products of powers of a minimal transformation, Journal d'Analyse Math. 64 (1994), 241-262. MR 96f:54047

Department of Mathematics, Beloit College, Beloit, Wisconsin 53511

E-mail address: ellis@beloit.edu

Department of Mathematics, University of Minnesota, Minneapolis, Minnesota 55455

E-mail address: robellis@math.umn.edu

Department of Mathematics, Rutgers University, Camden, New Jersey 08102

E-mail address: nmahesh@crab.rutgers.edu 